\title{
COHOMOLOGY GROUPS INVARIANT UNDER CONTINUOUS ORBIT EQUIVALENCE
}

\author{
YONGLE JIANG
}

\begin{abstract}
By the work of Brodzki-Niblo-Nowak-Wright and Monod, topological amenability of a continuous group action can be characterized using uniformly finite homology groups or bounded cohomology groups associated to this action. We show that (certain variations of) these groups are invariants for topologically free actions under continuous orbit equivalence.
\end{abstract}

\section{INTRODUCTION}

We continue our study of continuous orbit equivalence introduced by Li [14]. In this paper, we focus on certain (co)homology groups for a continuous group action. Let us first review the introduction of these (co)homology groups.

Let $G$ be a countable discrete group. There are two remarkable characterizations of amenablility of $G$. One, given by Johnson-Ringrose [13], says that $G$ is amenable if and only if the first bounded cohomology group with coefficients in $\ell_{0}^{1}(G)^{* *}$ vanishes, i.e. $H_{b}^{1}\left(G, \ell_{0}^{1}(G)^{* *}\right)=0$, where $\ell_{0}^{1}(G)$ denotes the augmentation ideal, i.e. kernel of the summation map from $\ell^{1}(G)$ to $\mathbb{R}$. By contrast, Block and Weinberger [2] described amenability in terms of non-vanishing of the 0-dimensional uniformly finite homology of $G$, i.e. $H_{0}^{u f}(G, \mathbb{R}) \neq 0$. For a short, unified proof of these two results, see [5]. In particular, it was observed there that $H_{*}^{u f}(G, \mathbb{R})=H_{*}\left(G, \ell^{\infty}(G)\right)$ if $G$ is finitely generated.

The notion of amenable actions of groups acting on topological spaces generalizes the concept of amenability and appears in many areas of mathematics. For example, a group acts amenably on a point if and only if it is amenable, while every hyperbolic group acts amenably on its Gromov boundary. For more on amenable actions, see $[1,10,11,18]$.

Parallel with these characterizations, people also found two similar characterizations for the amenablity of group actions in the topological sense. To do this, the key step is to find appropriate coefficient modules associated to a continuous action $G \curvearrowright X$.

More precisely, Brodzki, Niblo, Nowak and Wright considered the standard module $W_{0}(G, X)$ and its submodule $N_{0}(G, X):=C\left(X, \ell_{0}^{1}(G)\right)$. Note that $W_{0}(G, X)^{*}$

Date: November 12, 2019.

Key words and phrases. Continuous orbit equivalence; uniformly finite homology; bounded cohomology. 
and $N_{0}(G, X)^{* *}$ reduce to $\ell^{\infty}(G)$ and $\ell_{0}^{1}(G)^{* *}$ respectively when $X$ is a point. Naturally, they considered the bounded cohomology groups with coefficients in $N_{0}(G, X)^{* *}$ [3] and also the uniformly finite homology of an action, $H_{n}^{u f}(G \curvearrowright X)$ as the group homology with coefficients in $W_{0}(G, X)^{*}$, i.e. $H_{n}^{u f}(G \curvearrowright X):=H_{n}\left(G, W_{0}(G, X)^{*}\right)$ [4]. They succeeded in characterizing amenability of actions using these (co)homology groups, generalizing the above results of Johson-Ringrose and Block-Weinberger for group case. A similar approach was also taken by Monod in [17].

In [2] (see also [15]), among other results, $H_{*}^{u f}(G, \mathbb{R})$ is shown to be an invariant for groups under coarse equivalence, i.e. quasi-isometry if the groups are finitely generated. Hence it is natural to ask whether the above (co)homology groups for actions are also invariants under some "coarse equivalence" for actions.

In this paper, we show this is indeed possible if we take "coarse equivalence" to be "continuous orbit equivalence", and actions are assumed to be topologically free.

Let us recall the definition of continuous orbit equivalence, for known results on this notion and its connection to geometric group theory, see [7-9, 12, 14, 15].

Let $G \curvearrowright X$ and $H \curvearrowright Y$ be two actions by homeomorphisms, where $G, H$ are countable discrete groups and $X, Y$ are compact Hausdorff spaces. Following [14], we say the two actions are continuous orbit equivalent (abbreviated as COE) if there are homeomorphisms $\phi: X \approx Y, \psi: Y \approx X$ and continuous maps $c: G \times X \rightarrow H$, $c^{\prime}: H \times Y \rightarrow G$ such that $\phi(g x)=c(g, x) \phi(x)$ and $\psi(h y)=c^{\prime}(h, y) \psi(y)$ hold for all $g \in G, h \in H, x \in X$ and $y \in Y$. If these two actions are topologically free, i.e. points with trivial stabilizers are dense, then both $c$ and $c^{\prime}$ are cocycles [14, Lemma 2.8]. Recall that $c: G \times X \rightarrow H$ is a cocycle if $c\left(g_{1} g_{2}, x\right)=c\left(g_{1}, g_{2} x\right) c\left(g_{2}, x\right)$ for all $g_{1}, g_{2} \in G$ and all $x \in X$.

Now, we can state our main theorems. Note that all acting groups are assumed to be countable discrete and spaces are assumed to be compact Hausdorff.

Theorem 1.1. Let $G \curvearrowright X$ and $H \curvearrowright Y$ be topologically free actions. If these two actions are COE, then $H_{0}^{u f}(G \curvearrowright X) \cong H_{0}^{u f}(H \curvearrowright Y)$ and $H_{n}\left(G, N_{0}(G, X)^{*}\right) \cong$ $H_{n}\left(H, N_{0}(H, Y)^{*}\right)$ for all $n \geq 0$.

Theorem 1.2. Let $G \curvearrowright X$ and $H \curvearrowright Y$ be topologically free actions. If these two actions are $C O E$, then $H_{b}^{p}\left(G, N_{0}(G, X)^{* *}\right) \cong H_{b}^{p}\left(H, N_{0}(H, Y)^{* *}\right)$ for all $p \geq 0$.

The paper is organized as follows.

Besides the introduction, the paper contains five other sections. In Section 2, we review the definitions of group (co)homology and certain coefficient modules. In Section 3, we state several lemmas on basic properties of the orbit cocycles and observe that the coefficient modules are closed under restriction maps. These are the crucial ingredients for constructing bijective (co)chain maps later. The next two sections will take up the majority of our paper: Section 4 proves Theorem 1.1 and Section 5 proves Theorem 1.2. The proof of these two theorems uses the same idea: we construct maps between (co)chain complexes directly and then use the whole sections to verify these maps are bijective (co)chain maps. Detailed proof for lower 
dimensional cases will be included to illustrate the main ideas. We conclude with several remarks in Section 6 to discuss corollaries of the theorems and other related questions.

Following the convention in $[3,4,17]$, all Banach spaces are assumed to be real.

\section{Preliminaries}

2.1. Group (co)homology. We briefly recall the definition of group homology and (bounded) group cohomology using bar resolutions, see [15, §4.3], [6, Chapter III, $\S 1]$ and [16].

Let $G$ be a group and $V$ be a $\mathbb{Z} G$-module. Let $\left(C_{*}(V), \partial_{*}\right)$ be the chain complex

$\ldots \stackrel{\partial_{3}}{\rightarrow} C_{2}(V) \stackrel{\partial_{2}}{\rightarrow} C_{1}(V) \stackrel{\partial_{7}}{\rightarrow} C_{0}(V)$ with $C_{0}(V)=V, C_{n}(V)=C_{f}\left(G^{n}, V\right)$, where $C_{f}$ stands for maps with finite support, and $\partial_{n}=\sum_{i=0}^{n}(-1)^{i} \partial_{n}^{(i)}$, where

$$
\begin{aligned}
\partial_{n}^{(0)}(f)\left(g_{1}, \ldots, g_{n-1}\right) & =\sum_{g_{0} \in G} g_{0}^{-1} f\left(g_{0}, g_{1}, \ldots, g_{n-1}\right), \\
\partial_{n}^{(i)}(f)\left(g_{1}, \ldots, g_{n-1}\right) & =\sum_{\substack{g, \bar{g} \in G \\
g \bar{g}=g_{i}}} f\left(g_{1}, \ldots, g_{i-1}, g, \bar{g}, g_{i+1}, g_{n-1}\right) \text { for } 1 \leq i \leq n-1, \\
\partial_{n}^{(n)}(f)\left(g_{1}, \ldots, g_{n-1}\right) & =\sum_{g_{n} \in G} f\left(g_{1}, \ldots, g_{n-1}, g_{n}\right) .
\end{aligned}
$$

Then we define $H_{n}(G, V):=\operatorname{Ker}\left(\partial_{n}\right) / \operatorname{Im}\left(\partial_{n+1}\right)$.

Let $\left(C^{*}(V), \partial^{*}\right)$ be the cochain complex $C^{0}(V) \stackrel{\partial^{0}}{\rightarrow} C^{1}(V) \stackrel{\partial^{1}}{\rightarrow} C^{2}(V) \stackrel{\partial^{2}}{\rightarrow} \ldots$ with $C^{0}(V)=V, C^{n}(V)=C\left(G^{n}, V\right)$ for all $n \geq 1$, and $\partial^{n}=\sum_{i=0}^{n+1}(-1)^{i} \partial_{(i)}^{n}$, where

$$
\begin{aligned}
\partial_{(0)}^{n}(f)\left(g_{0}, \ldots, g_{n}\right) & =g_{0} f\left(g_{1}, \ldots, g_{n}\right), \\
\partial_{(i)}^{n}(f)\left(g_{0}, \ldots, g_{n}\right) & =f\left(g_{0}, \cdots, g_{i-1} g_{i}, \ldots, g_{n}\right) \text { for } 1 \leq i \leq n, \\
\partial_{(n+1)}^{n}(f)\left(g_{0}, \ldots, g_{n}\right) & =f\left(g_{0}, \ldots, g_{n-1}\right) .
\end{aligned}
$$

Then we define $H^{n}(G, V):=\operatorname{Ker}\left(\partial^{n}\right) / \operatorname{Im}\left(\partial^{n-1}\right)$.

If $V$ is a Banach $G$-module, we may replace $C^{*}(V)$ by $C_{b}^{*}(V)$, where $C_{b}^{n}(V):=$ $C_{b}\left(G^{n}, V\right)$ and $C_{b}$ stands for uniformly bounded maps, then the group cohomology we get is the bounded cohomology group, written as $H_{b}^{n}(G, V)$.

2.2. Coefficient modules. The module $V$ we are interested in are certain dual or double dual Banach spaces, i.e. $N_{0}(G, X)^{*}, W_{0}(G, X)^{*}$ and $N_{0}(G, X)^{* *}$ associated to a continuous action $G \curvearrowright X$. Let us recall their definition below $[3,4]$.

The space $C\left(X, \ell^{1}(G)\right)$ of continuous $\ell^{1}(G)$-valued functions on $X$ is equipped with the sup- $\ell^{1}$ norm

$$
\|\xi\|=\sup _{x \in X} \sum_{g \in G}\left|\xi_{x}(g)\right|
$$

Here, for $\xi \in C\left(X, \ell^{1}(G)\right)$, we write $\xi_{x}(g):=\xi(x)(g)$. We remind the reader that we are using a different notation from the one in $[3,4]$, where $\xi_{g}(x)$ was used. 
In this notation, the Banach space $C\left(X, \ell^{1}(G)\right)$ is equipped with a natural action of $G$,

$$
(g \cdot \xi)_{x}(h):=\xi_{g^{-1} x}\left(g^{-1} h\right)
$$

for each $g, h \in G$ and $x \in X$.

The summation map on $\ell^{1}(G)$ induces a continuous map $\sigma: C\left(X, \ell^{1}(G)\right) \rightarrow$ $C(X)$, where $C(X)$ is equipped with the $\ell^{\infty}$ norm. The space $N_{0}(G, X)$ is defined to be the pre-image $\sigma^{-1}(0)$ which we identify as $C\left(X, \ell_{0}^{1}(G)\right)$. Then we define $W_{0}(G, X):=\sigma^{-1}(\mathbb{R})$, where $\mathbb{R}$ is regarded as constant functions on $X$. Note that $W_{0}(G, X)=N_{0}(G, X) \oplus \mathbb{R}$, where $\mathbb{R}$ is identified as constant $\mathbb{R} \delta_{e}$-valued functions on $X$, here $e$ is the neutral element in $G$. Obviously, both $N_{0}(G, X)$ and $W_{0}(G, X)$ are invariant under the above $G$-action since $\sigma$ is $G$-equivariant. Also recall that if $V$ is a Banach $G$-module, then the Banach dual space $V^{*}$ is also equipped with a $G$-module structure by requiring $(g \phi)(\xi)=\phi\left(g^{-1} \xi\right)$ for $g \in G, \phi \in V^{*}$ and $\xi \in V$.

\section{Properties of orbit COCYCles AND RESTRICTION INVARIANT PROPERTy}

In this section, we state several lemmas proving basic properties of orbit cocycles and observe that the module $N_{0}(G, X)$ is restriction invariant. These give us the hint on how to define bijective (co)chain maps between two (co)chain complexes later.

From now on, we use the notations introduced when defining COE, i.e. $\phi: X \approx Y$ and $\psi: Y \approx X$ are homeomorphisms; $c: G \times X \rightarrow H$ and $c^{\prime}: H \times Y \rightarrow G$ are maps satisfying certain identities. Concerning COE between topologically free actions, the following property will be used frequently.

Lemma 3.1. [14, Lemma 2.10] Let $G \curvearrowright X$ and $H \curvearrowright Y$ be topologically free actions that are COE. Then $c^{\prime}(c(g, x), \phi(x))=g$ for all $g \in G, x \in X$. Similarly, $c\left(c^{\prime}(h, y), \psi(y)\right)=h$ for all $h \in H, y \in Y$.

Clearly, this implies that for all $x \in X, G \ni g \mapsto c(g, x) \in H$ is a bijection.

The starting point for our proof is the following lemma.

Lemma 3.2. Let $G \curvearrowright X$ and $H \curvearrowright Y$ be topologically free actions which are COE. Then there exists a (linear) map $\pi$ from $C\left(Y, \ell^{1}(H)\right)$ onto $C\left(X, \ell^{1}(G)\right)$ which is an isometry, and which moreover has the following properties: $\pi\left(W_{0}(H, Y)\right)=$ $W_{0}(G, X), \pi\left(N_{0}(H, Y)\right)=N_{0}(G, X)$ and $\pi(\mathbb{R})=\mathbb{R}$, where $\mathbb{R}$ is identified as constant $\mathbb{R} \delta_{e}$-valued functions on $X$.

Proof. We define $\pi: C\left(Y, \ell^{1}(H)\right) \rightarrow C\left(X, \ell^{1}(G)\right)$ by setting $\pi(\xi)=\xi^{\prime}$, where

$$
\xi_{x}^{\prime}(g):=\xi_{\phi(x)}\left(c\left(g, g^{-1} x\right)\right) .
$$

First, we check $\pi$ is a well-defined isometry.

Note that for all $x \in X, G \ni g \mapsto c\left(g^{-1}, x\right)^{-1}=c\left(g, g^{-1} x\right) \in H$ is a bijection. A calculation shows that $\sum_{g} \xi_{x}^{\prime}(g)=\sum_{h} \xi_{\phi(x)}(h)$ for all $x \in X$ and hence $\|\xi\|=\left\|\xi^{\prime}\right\|$. So $\pi$ is an isometry. 
Now we check $\xi^{\prime} \in C\left(X, \ell^{1}(G)\right)$. For any given $x \in X$ and $\epsilon>0$, we need to find $\mathcal{U}_{x}$, a neighborhood of $x$, such that if $x^{\prime} \in \mathcal{U}_{x}$, then $\sum_{g \in G}\left|\xi_{x}^{\prime}(g)-\xi_{x^{\prime}}^{\prime}(g)\right|<\epsilon$.

Since $\xi \in C\left(Y, \ell^{1}(H)\right)$, there exists a small neighborhood $\mathcal{V}_{\phi(x)}$ containing $\phi(x)$ such that if $y^{\prime} \in \mathcal{V}_{\phi(x)}$, then $\sum_{h \in H}\left|\xi_{y^{\prime}}(h)-\xi_{\phi(x)}(h)\right|<\epsilon / 4$.

Take a large finite set $F \subseteq H$ such that $\sum_{h \notin F}\left|\xi_{\phi(x)}(h)\right|<\epsilon / 4$. This implies that if $y^{\prime} \in \mathcal{V}_{\phi(x)}$, then

$$
\sum_{h \notin F}\left|\xi_{y^{\prime}}(h)\right| \leq \sum_{h \notin F}\left|\xi_{y^{\prime}}(h)-\xi_{\phi(x)}(h)\right|+\sum_{h \notin F}\left|\xi_{\phi(x)}(h)\right| \leq \epsilon / 2 .
$$

Now, we take $\mathcal{U}_{x}$ to be any sufficiently small neighborhood of $x$, which is contained in $\psi\left(\mathcal{V}_{\phi(x)}\right)$ and satisfies the property that for any $x^{\prime} \in \mathcal{U}_{x}$, we have $c\left(g^{-1}, x\right)=$ $c\left(g^{-1}, x^{\prime}\right)$ for all $g \in K$, where $K:=\left\{g \in G: c\left(g^{-1}, x\right)^{-1} \in F\right\}$ is a finite set.

Take any $x^{\prime} \in \mathcal{U}_{x}$, note that $g \in K$ iff $c\left(g^{-1}, x\right)^{-1} \in F$ iff $c\left(g^{-1}, x^{\prime}\right)^{-1} \in F$. Moreover, $x^{\prime} \in \mathcal{U}_{x}$ implies $\phi\left(x^{\prime}\right) \in \mathcal{V}_{\phi(x)}$. Hence,

$$
\begin{aligned}
\sum_{g \in G}\left|\xi_{x}^{\prime}(g)-\xi_{x^{\prime}}^{\prime}(g)\right| & =\sum_{g \in K}\left|\xi_{x}^{\prime}(g)-\xi_{x^{\prime}}^{\prime}(g)\right|+\sum_{g \notin K}\left|\xi_{x}^{\prime}(g)-\xi_{x^{\prime}}^{\prime}(g)\right| \\
& \leq \sum_{h \in F}\left|\xi_{\phi(x)}(h)-\xi_{\phi\left(x^{\prime}\right)}(h)\right|+\sum_{h \notin F}\left|\xi_{\phi(x)}(h)\right|+\sum_{h \notin F}\left|\xi_{\phi\left(x^{\prime}\right)}(h)\right| \\
& \leq \epsilon / 4+\epsilon / 4+\epsilon / 2=\epsilon .
\end{aligned}
$$

Second, it is easy to check $\pi\left(W_{0}(H, Y)\right) \subseteq W_{0}(G, X), \pi\left(N_{0}(H, Y)\right) \subseteq N_{0}(G, X)$ and $\pi(\mathbb{R}) \subseteq \mathbb{R}$ hold. To see $\subseteq$ is really $=$, we need to find the inverse of $\pi$.

By symmetry, we can define another isometry $L: C\left(X, \ell^{1}(G)\right) \rightarrow C\left(Y, \ell^{1}(H)\right)$ by setting $L(\eta)=\eta^{\prime}$, where

$$
\eta_{y}^{\prime}(h):=\eta_{\psi(y)}\left(c^{\prime}\left(h, h^{-1} y\right)\right)
$$

It is straightforward to check that $L$ is the inverse of $\pi$. Indeed, this boils down to check that $c\left(c^{\prime}(h, y), \psi(y)\right)=h$ and $c^{\prime}(c(g, x), \phi(x))=g$, which hold by Lemma 3.1. Then using $L$, we know $\pi$ maps the three subspaces onto the corresponding ones.

Observation. In the proof of the main theorems, we will use the following observation, which shows that $N_{0}(G, X)$ is restriction invariant.

Under the assumptions in the main theorems, for any given $g \in G$, let us list the elements in the finite set $c\left(g^{-1}, X\right):=\left\{c\left(g^{-1}, x\right) \mid x \in X\right\}$ as $h_{1}^{-1}, \ldots, h_{n}^{-1}$. Then define $X_{i}:=\left\{x \in X: c\left(g^{-1}, x\right)=h_{i}^{-1}\right\}$. Clearly, $X=\sqcup_{i} X_{i}$ and each $X_{i}$ is clopen since $c$ is continuous. Note that $N_{0}(G, X)=C\left(X, \ell_{0}^{1}(G)\right)=\oplus_{i} C\left(X_{i}, \ell_{0}^{1}(G)\right)$. For each $\xi^{\prime} \in C\left(X, \ell_{0}^{1}(G)\right)$, let $\left.\xi^{\prime}\right|_{X_{i}}$ be the $i$-th component with respect to the above decomposition, i.e. $\left(\left.\xi^{\prime}\right|_{X_{i}}\right)_{g}(x)=\xi_{g}^{\prime}(x)$ if $x \in X_{i}$ and zero otherwise. Clearly, $\left.\xi^{\prime}\right|_{X_{i}} \in C\left(X, \ell_{0}^{1}(G)\right)$. For each $\tau \in N_{0}(G, X)^{*}$, define $\tau_{i} \in N_{0}(G, X)^{*}$ by setting $\tau_{i}\left(\xi^{\prime}\right)=\tau\left(\left.\xi^{\prime}\right|_{X_{i}}\right)$. Then, we have that $\xi^{\prime}=\left.\sum_{i} \xi^{\prime}\right|_{X_{i}}$ and $\tau=\sum_{i} \tau_{i}$. Note that the above decomposition is not $G$-equivariant, i.e. $g \tau_{i} \neq(g \tau)_{i}$. Moreover, for any clopen subset $X_{0}$ of $X,\left.\xi\right|_{X_{0}} \in N_{0}(G, X)$ if $\xi \in N_{0}(G, X)$, hence $\left.\tau\right|_{X_{0}}$ is also well-defined. (By convention, $\left.\xi^{\prime}\right|_{\emptyset}:=0$ and $\left.\tau\right|_{\emptyset}:=0$.) 
Below we record two more lemmas that we use frequently later to simplify the proof. The first lemma tells us the map $\pi$ is equivariant in a local sense, and the second one tells us how the composition of $\pi$ and $L$ behaves under restrictions.

Lemma 3.3. Let $g \in G, h \in H$ and $\xi \in N_{0}(H, Y)$. If $X_{0} \subseteq\left\{x \in X: c\left(g^{-1}, x\right)=\right.$ $\left.h^{-1}\right\}$ is clopen, then $\left.(\pi(h \xi))\right|_{X_{0}}=\left.(g \pi(\xi))\right|_{X_{0}}=g\left(\left.\pi(\xi)\right|_{g^{-1} X_{0}}\right)$, where $\pi$ is the map constructed in Lemma 3.2.

Proof. We may assume $X_{0} \neq \emptyset$; otherwise, the equality holds trivially as both side equals zero. Take any $x \in X$ and $g^{\prime} \in G$, we get

$$
\begin{aligned}
{\left[\left.(\pi(h \xi))\right|_{X_{0}}\right]_{x}\left(g^{\prime}\right) } & =\left\{\begin{array}{l}
(h \xi)_{\phi(x)}\left(c\left(g^{\prime}, g^{-1} x\right)\right), \text { if } x \in X_{0} \\
0, \text { otherwise }
\end{array}\right. \\
& =\left\{\begin{array}{l}
\xi_{h^{-1} \phi(x)}\left(h^{-1} c\left(g^{\prime}, g^{\prime-1} x\right)\right), \text { if } x \in X_{0} \\
0, \text { otherwise }
\end{array}\right. \\
& =\left\{\begin{array}{l}
\xi_{\phi\left(g^{-1} x\right)}\left(c\left(g^{-1} g^{\prime}, g^{\prime-1} x\right)\right), \text { if } x \in X_{0} \\
0, \text { otherwise }
\end{array}\right. \\
& =\left\{\begin{array}{l}
{[\pi(\xi)]_{g^{-1} x}\left(g^{-1} g^{\prime}\right), \text { if } x \in X_{0}} \\
0, \text { otherwise }
\end{array}\right. \\
& =\left\{\begin{array}{l}
{[g(\pi(\xi))]_{x}\left(g^{\prime}\right), \text { if } x \in X_{0}} \\
0, \text { otherwise }
\end{array}\right. \\
& =\left[\begin{array}{l}
\left.\left.(g \pi(\xi))\right|_{X_{0}}\right]_{x}\left(g^{\prime}\right) .
\end{array}\right.
\end{aligned}
$$

Hence, $\left.\pi(h \xi)\right|_{X_{0}}=\left.(g \pi(\xi))\right|_{X_{0}}$ holds. It is easy to check $\left.(g \pi(\xi))\right|_{X_{0}}=g\left(\left.\pi(\xi)\right|_{g^{-1} X_{0}}\right)$ holds by definition.

Lemma 3.4. Let $X_{0}$ and $Y_{0}$ be clopen subsets of $X$ and $Y$ respectively. If $\eta \in$ $N_{0}(G, X)$ and $\xi \in N_{0}(H, Y)$, then $\left.\pi\left(\left.L(\eta)\right|_{Y_{0}}\right)\right|_{X_{0}}=\left.\eta\right|_{X_{0} \cap \psi\left(Y_{0}\right)}$ and $\left.L\left(\left.\pi(\xi)\right|_{X_{0}}\right)\right|_{Y_{0}}=$ $\left.\xi\right|_{Y_{0} \cap \phi\left(X_{0}\right)}$.

Proof. We will check the first equality below, the second one can be checked similarly.

Let $\xi=\left.L(\eta)\right|_{Y_{0}} \in N_{0}(H, Y)$. Take any $g \in G$ and $x \in X$, then

$$
\begin{aligned}
& \left(\left.\pi(\xi)\right|_{X_{0}}\right)_{x}(g) \\
= & \begin{cases}\xi_{\phi(x)}\left(c\left(g, g^{-1} x\right)\right), & \text { if } x \in X_{0} \\
0, & \text { otherwise }\end{cases}
\end{aligned}
$$

(def. of restriction map and $\pi$ )

$$
= \begin{cases}\eta_{x}\left(c^{\prime}\left(c\left(g, g^{-1} x\right), c\left(g, g^{-1} x\right)^{-1} \phi(x)\right)\right), & \text { if } x \in X_{0} \cap \psi\left(Y_{0}\right) \\ 0, & \text { otherwise }\end{cases}
$$

(def. of $\xi$ and $L$ ) 
$=\left\{\begin{array}{l}\eta_{x}(g), \quad \text { if } x \in X_{0} \cap \psi\left(Y_{0}\right) \\ 0, \quad \text { otherwise }\end{array}\right.$

(cocycle identity and Lemma 3.1)

$=\left(\left.\eta\right|_{X_{0} \cap \psi\left(Y_{0}\right)}\right)_{x}(g)$.

Hence, $\left.\left(\pi\left(\left.L(\eta)\right|_{Y_{0}}\right)\right)\right|_{X_{0}}=\left.\eta\right|_{X_{0} \cap \psi\left(Y_{0}\right)}$.

Notations. Here we record some notations used in the context.

- $\phi: X \approx Y$ and $\psi: Y \rightarrow X$ are the homemophisms in the definition of COE.

- $c: G \times X \rightarrow H$ and $c^{\prime}: H \times Y \rightarrow X$ denote the orbit cocycles.

- $\pi$ is the isometry from $N_{0}(H, Y)$ to $N_{0}(G, X)$ as defined in Lemma 3.2.

- $L$ is the inverse map of $\pi$ as defined in Lemma 3.2.

- $c(g, X)$ denotes the finite set $\{c(g, x): x \in X\}$.

- $c^{\prime}(h, Y)$ denotes the finite set $\left\{c^{\prime}(h, y): y \in Y\right\}$.

- $X_{g, h}$ denotes the set $\left\{x \in X: c\left(g^{-1}, x\right)=h^{-1}\right\}$. For later use, we usually simplify $X_{g_{i}, h}$ (resp. $X_{g, h_{j}}$ ) to $X_{i}$ (resp. $X_{j}$ ) if the variable $h($ resp. $g$ ) is fixed or clear from the context.

- $\eta$ denotes an element in $N_{0}(G, X)$ or $N_{0}(G, X)^{* *}$ depending on the context.

- $\xi$ denotes an element in $N_{0}(H, Y)$ or $N_{0}(H, Y)^{* *}$ depending on the context.

- $\tau$ denotes an element in $N_{0}(G, X)^{*}$.

- $\nu$ denotes an element in $N_{0}(H, Y)^{*}$.

\section{Proof of Theorem 1.1}

By [4, Corollary 10], we know that $H_{0}^{u f}(G \curvearrowright X) \cong \mathbb{R} \oplus H_{0}\left(G, N_{0}(G, X)^{*}\right)$ or $H_{0}\left(G, N_{0}(G, X)^{*}\right)$ depending on whether the action is amenable or not. Therefore, to prove Theorem 1.1, it suffices to prove the second part, i.e. $H_{n}\left(G, N_{0}(G, X)^{*}\right) \cong$ $H_{n}\left(H, N_{0}(H, Y)^{*}\right)$ for all $n \geq 0$.

We will construct a (linear) map $S_{n}: C_{f}\left(G^{n}, N_{0}(G, X)^{*}\right) \rightarrow C_{f}\left(H^{n}, N_{0}(H, Y)^{*}\right)$ and check $\partial_{n} S_{n}=S_{n-1} \partial_{n}$ for each $n$, i.e. $S_{n}$ is a chain map. Moreover, we will show it is an isomorphism by finding the inverse map $T_{n}$. Clearly, this will induce an isomorphism between homology groups and hence finish the proof.

We present the detailed proof when $n=0,1$ to illustrate the main ideas. The proof of the general case will be given after the proof of these two cases.

4.1. Case $n=0$. Clearly, the map $\pi: N_{0}(H, Y) \rightarrow N_{0}(G, X)$ as used in Lemma 3.2 induces a map, denoted by $\pi^{*}$, on the dual spaces.

Define $S_{0}:=\pi^{*}$.

Let $f \in C_{f}\left(G, N_{0}(G, X)^{*}\right)$, we define $S_{1}(f) \in C_{f}\left(H, N_{0}(H, Y)^{*}\right)$ by setting $S_{1}(f)=$ $f^{\prime}$, where

$$
f^{\prime}\left(h_{1}\right)(\xi):=\sum_{j} f\left(g_{j}\right)\left(\left.\pi(\xi)\right|_{X_{j}}\right)
$$


Here $\xi \in N_{0}(H, Y), c^{\prime}\left(h_{1}, Y\right):=\left\{c^{\prime}\left(h_{1}, y\right): y \in Y\right\}:=\left\{g_{j} \mid j\right\}, X_{j}=\{x \in$ $\left.X: c\left(g_{j}^{-1}, x\right)=h_{1}^{-1}\right\}$. Strictly speaking, it is better to use notation $X_{g_{j}, h_{1}}$ for $X_{j}$, but we often simplify the subscripts if no confusion arises.

Clearly, $S_{0}, S_{1}$ are well-defined. Indeed, to see $S_{1}$ is well-defined, observe that if $\operatorname{supp}(f)=F$, then $\operatorname{supp}\left(S_{1}(f)\right) \subseteq \cup_{g \in F} c(g, X)$, which is finite once $F$ is a finite set.

Step 1: we check $\partial_{1} S_{1}=S_{0} \partial_{1}$.

Let $f \in C_{f}\left(G, N_{0}(G, X)^{*}\right)$ and $f^{\prime}=S_{1}(f)$. Recall $\partial_{1}\left(f^{\prime}\right)=\sum_{h_{1} \in H} h_{1}^{-1} f^{\prime}\left(h_{1}\right)-$ $\sum h_{1} \in H f^{\prime}\left(h_{1}\right)$. We need to show $\partial_{1} S_{1}(f)=S_{0} \partial_{1}(f)$; equivalently,

$$
\sum_{h_{1} \in H} h_{1}^{-1} f^{\prime}\left(h_{1}\right)-\sum_{h_{1} \in H} f^{\prime}\left(h_{1}\right)=\sum_{g \in G} \pi^{*}\left(g^{-1} f(g)\right)-\sum_{g \in G} \pi^{*}(f(g)) .
$$

It suffices to check

$$
\begin{aligned}
\sum_{h_{1} \in H} h_{1}^{-1} f^{\prime}\left(h_{1}\right) & =\sum_{g \in G} \pi^{*}\left(g^{-1} f(g)\right), \\
\sum_{h_{1} \in H} f^{\prime}\left(h_{1}\right) & =\sum_{g \in G} \pi^{*}(f(g)) .
\end{aligned}
$$

To check (2), take any $\xi \in N_{0}(H, Y)$, then

$$
\begin{aligned}
\sum_{h_{1}} f^{\prime}\left(h_{1}\right)(\xi) & =\sum_{h_{1}} \sum_{j} f\left(g_{j}\right)\left(\left.\pi(\xi)\right|_{X_{j}}\right)=\sum_{g} \sum_{h_{1}, h_{1} \in c(g, X)} f(g)\left(\left.\pi(\xi)\right|_{X_{g, h_{1}}}\right) \\
& =\sum_{g} f(g)(\pi(\xi))=\sum_{g} \pi^{*}(f(g))(\xi) .
\end{aligned}
$$

The 2nd last equality holds since $X=\sqcup_{h_{1}, h_{1} \in c(g, X)} X_{g, h_{1}}$ for every $g$.

Similarly, let us check (1) holds below.

$$
\begin{aligned}
\sum_{h_{1}}\left(h_{1}^{-1} f^{\prime}\left(h_{1}\right)\right)(\xi) & =\sum_{h_{1}} f^{\prime}\left(h_{1}\right)\left(h_{1} \xi\right)=\sum_{h_{1}} \sum_{j} f\left(g_{j}\right)\left(\left.\pi\left(h_{1} \xi\right)\right|_{X_{j}}\right) \\
& =\sum_{g} \sum_{h_{1}, h_{1} \in c(g, X)} f(g)\left(\left.\pi\left(h_{1} \xi\right)\right|_{X_{g, h_{1}}}\right) \\
& =\sum_{g} \sum_{h_{1}, h_{1} \in c(g, X)} f(g)\left(\left.(g \pi(\xi))\right|_{X_{g, h_{1}}}\right)(\text { by Lemma 3.3) } \\
& =\sum_{g} f(g)\left(\left.(g \pi(\xi))\right|_{\left.\sqcup_{h_{1} \in c(g, X)} X_{g, h_{1}}\right)}\right. \\
& =\sum_{g} f(g)(g \pi(\xi))\left(\operatorname{as} X=\sqcup_{h_{1} \in c(g, X)} X_{g, h_{1}}\right) \\
& =\sum_{g} \pi^{* *}\left(g^{-1} f(g)\right)(\xi) .
\end{aligned}
$$

Step 2: $S_{0}$ is an isomorphism. 
Recall in the proof of Lemma 3.2, there exists some map $L: N_{0}(G, X) \rightarrow N_{0}(H, Y)$ defined by setting $L(\eta)=\eta^{\prime}$, where $\eta_{h}^{\prime}(y):=\eta_{\psi(y)}\left(c^{\prime}\left(h, h^{-1} y\right)\right)$. By symmetry, it also induces a well-defined map $T_{0}:=L^{*}: N_{0}(H, Y)^{*} \rightarrow N_{0}(G, X)^{*}$. Clearly, $\pi^{*} L^{*}=i d$ and $L^{*} \pi^{*}=i d$.

4.2. Case $n=1$. Write $c^{\prime}\left(h_{0}, Y\right)=\left\{g_{i} \mid i\right\}, c^{\prime}\left(h_{1}, Y\right)=\left\{g_{j} \mid j\right\}, c^{\prime}\left(h_{2}, Y\right)=\left\{g_{k} \mid k\right\}$, $c^{\prime}(h, Y)=\left\{g_{s} \mid s\right\}$ and $c^{\prime}(\bar{h}, Y)=\left\{g_{t} \mid t\right\}$. In this subsection, we will use the following notations.

$$
\begin{array}{ll}
X_{i}:=\left\{x: c\left(g_{i}^{-1}, x\right)=h_{0}^{-1}\right\}, & X_{j}:=\left\{x: c\left(g_{j}^{-1}, x\right)=h_{1}^{-1}\right\}, \\
X_{k}:=\left\{x: c\left(g_{k}^{-1}, x\right)=h_{2}^{-1}\right\}, & X_{s}:=\left\{x: c\left(g_{s}^{-1}, x\right)=h^{-1}\right\}, \\
X_{t}:=\left\{x: c\left(g_{t}^{-1}, x\right)=\bar{h}^{-1}\right\} . &
\end{array}
$$

Strictly speaking, it is better to use the notation $X_{g_{i}, h_{0}}, X_{g_{j}, h_{1}}, X_{g_{k}, h_{2}}, X_{g_{s}, h}, X_{g_{t}, \bar{h}}$ for $X_{i}, X_{j}, X_{k}, X_{s}, X_{t}$ respectively, but we often simplify the subscripts as the variables $h_{0}, h_{1}, h_{2}, h, \bar{h}$ are usually fixed in the context.

Let $\theta \in C_{f}\left(G^{2}, N_{0}(G, X)^{*}\right)$, we define $S_{2}: C_{f}\left(G^{2}, N_{0}(G, X)^{*}\right) \rightarrow C_{f}\left(H^{2}, N_{0}(H, Y)^{*}\right)$ by setting $\theta^{\prime}=S_{2} \theta$, where for any $\xi \in N_{0}(H, Y)$,

$$
\theta^{\prime}\left(h_{0}, h_{1}\right)(\xi):=\sum_{i, j} \theta\left(g_{i}, g_{j}\right)\left(\left.\pi(\xi)\right|_{g_{i} X_{j} \cap X_{i}}\right) .
$$

Note that $S_{2}$ is well-defined. Indeed, observe that

$$
\operatorname{supp}\left(\theta^{\prime}\right) \subseteq \bigcup_{\left(g_{i}, g_{j}\right) \in \operatorname{supp}(\theta)} c\left(g_{i}, X\right) \times c\left(g_{j}, X\right) .
$$

Therefore, $\operatorname{supp}(\theta)$ is finite implies $\operatorname{supp}\left(\theta^{\prime}\right)$ is finite.

Write $f=\partial_{2} \theta$ and $f^{\prime}=S_{1} f$, from the definition of $\partial_{2}$, we know

$$
f\left(g_{1}\right)=\sum_{g_{0}} g_{0}^{-1} \theta\left(g_{0}, g_{1}\right)-\sum_{g, \bar{g}, g \bar{g}=g_{1}} \theta(g, \bar{g})+\sum_{g_{2}} \theta\left(g_{1}, g_{2}\right) .
$$

Step 1: we check $S_{1} \partial_{2}=\partial_{2} S_{2}$.

Evaluate both sides at $\theta$ and use the above notations, we are left to show the following identity holds.

$$
f^{\prime}\left(h_{1}\right)=\sum_{h_{0}} h_{0}^{-1} \theta^{\prime}\left(h_{0}, h_{1}\right)-\sum_{\substack{h, \bar{h} \\ h \bar{h}=h_{1}}} \theta^{\prime}(h, \bar{h})+\sum_{h_{2}} \theta^{\prime}\left(h_{1}, h_{2}\right) .
$$

Let $\xi \in N_{0}(H, Y)$, a calculation shows:

$$
\begin{aligned}
& f^{\prime}\left(h_{1}\right)(\xi) \\
= & \sum_{j} f\left(g_{j}\right)\left(\left.\pi(\xi)\right|_{X_{j}}\right) \\
= & \sum_{j}\left[\sum_{g} \theta\left(g, g_{j}\right)\left(g\left(\left.\pi(\xi)\right|_{X_{j}}\right)\right)-\sum_{\substack{g, \bar{g} \\
g \bar{g}=g_{j}}} \theta(g, \bar{g})\left(\left.\pi(\xi)\right|_{X_{j}}\right)+\sum_{g} \theta\left(g_{j}, g\right)\left(\left.\pi(\xi)\right|_{X_{j}}\right)\right] .
\end{aligned}
$$




$$
\begin{aligned}
& {\left[\sum_{h_{0}} h_{0}^{-1} \theta^{\prime}\left(h_{0}, h_{1}\right)-\sum_{\substack{h, \bar{h} \\
h \bar{h}=h_{1}}} \theta^{\prime}(h, \bar{h})+\sum_{h_{2}} \theta^{\prime}\left(h_{1}, h_{2}\right)\right](\xi) } \\
= & \sum_{h_{0}} \theta^{\prime}\left(h_{0}, h_{1}\right)\left(h_{0} \xi\right)-\sum_{\substack{h, \bar{h} \\
h \bar{h}=h_{1}}} \theta^{\prime}(h, \bar{h})(\xi)+\sum_{h_{2}} \theta^{\prime}\left(h_{1}, h_{2}\right)(\xi) \\
= & \sum_{h_{0}} \sum_{i, j} \theta\left(g_{i}, g_{j}\right)\left(\left.\pi\left(h_{0} \xi\right)\right|_{g_{i} X_{j} \cap X_{i}}\right)-\sum_{\substack{h, \bar{h} \\
h \bar{h}=h_{1}}} \sum_{s, t} \theta\left(g_{s}, g_{t}\right)\left(\left.\pi(\xi)\right|_{g_{s} X_{t} \cap X_{s}}\right) \\
& +\sum_{h_{2}} \sum_{j, k} \theta\left(g_{j}, g_{k}\right)\left(\left.\pi(\xi)\right|_{g_{j} X_{k} \cap X_{j}}\right) .
\end{aligned}
$$

Comparing the two expressions above, we just need to prove the corresponding terms are equal, i.e.

$$
\begin{aligned}
\sum_{j} \sum_{g} \theta\left(g, g_{j}\right)\left(g\left(\left.\pi(\xi)\right|_{X_{j}}\right)\right) & =\sum_{h_{0}} \sum_{i, j} \theta\left(g_{i}, g_{j}\right)\left(\left.\pi\left(h_{0} \xi\right)\right|_{g_{i} X_{j} \cap X_{i}}\right), \\
\sum_{j} \sum_{\substack{g, \bar{g} \\
g \bar{g}=g_{j}}} \theta(g, \bar{g})\left(\left.\pi(\xi)\right|_{X_{j}}\right) & =\sum_{\substack{h, \bar{h} \\
h \bar{h}=h_{1}}} \sum_{s, t} \theta\left(g_{s}, g_{t}\right)\left(\left.\pi(\xi)\right|_{g_{s} X_{t} \cap X_{s}}\right), \\
\sum_{j} \sum_{g} \theta\left(g_{j}, g\right)\left(\left.\pi(\xi)\right|_{X_{j}}\right) & =\sum_{h_{2}} \sum_{j, k} \theta\left(g_{j}, g_{k}\right)\left(\left.\pi(\xi)\right|_{g_{j} X_{k} \cap X_{j}}\right) .
\end{aligned}
$$

To check (3), apply Lemma 3.3 to get $\left.\pi\left(h_{0} \xi\right)\right|_{g_{i} X_{j} \cap X_{i}}=g_{i}\left(\left.\pi(\xi)\right|_{X_{j} \cap g_{i}^{-1} X_{i}}\right)$.

Then, RHS of $(3)=\sum_{j} \sum_{h_{0}, i} \theta\left(g_{i}, g_{j}\right)\left(g_{i}\left(\left.\pi(\xi)\right|_{X_{j} \cap g_{i}^{-1} X_{i}}\right)\right)$.

Now it suffices to prove for every $j$,

$$
\sum_{h_{0}, i} \theta\left(g_{i}, g_{j}\right)\left(g_{i}\left(\left.\pi(\xi)\right|_{X_{j} \cap g_{i}^{-1} X_{i}}\right)\right)=\sum_{g} \theta\left(g, g_{j}\right)\left(g\left(\left.\pi(\xi)\right|_{X_{j}}\right)\right) .
$$

First, recall that $g_{i}^{-1} X_{i}=\left\{x: c\left(g_{i}, x\right)=h_{0}\right\}$, which we denote by $Z_{g_{i}, h_{0}}$. Clearly, for every $g \in G, X=\sqcup_{h_{0} \in c(g, X)} Z_{g, h_{0}}$, so

$$
\begin{aligned}
\sum_{h_{0}, i} \theta\left(g_{i}, g_{j}\right)\left(g_{i}\left(\left.\pi(\xi)\right|_{X_{j} \cap g_{i}^{-1} X_{i}}\right)\right) & =\sum_{g} \sum_{h_{0} \in c(g, X)} \theta\left(g, g_{j}\right)\left(g\left(\left.\pi(\xi)\right|_{X_{j} \cap Z_{g, h_{0}}}\right)\right) \\
& =\sum_{g} \theta\left(g, g_{j}\right)\left(g\left(\left.\pi(\xi)\right|_{X_{j}}\right)\right) .
\end{aligned}
$$

To check (4), first, observe that we have a bijection/reordering between the two index sets: 


$$
\left\{\begin{array}{l|l}
\left(h, \bar{h}, g_{s}, g_{t}\right) & \begin{array}{l}
h \bar{h}=h_{1} \\
g_{s} \in c^{\prime}(h, Y), g_{t} \in c^{\prime}(\bar{h}, Y) \\
g_{s} X_{g_{t}, \bar{h}} \cap X_{g_{s}, h} \neq \emptyset
\end{array}
\end{array}\right\}
$$

and

$$
\left\{\begin{array}{l|l}
\left(g_{j}, g, \bar{g}, h, \bar{h}\right) & \begin{array}{l}
h \bar{h}=h_{1}, g \bar{g}=g_{j} \\
g_{j} \in c^{\prime}\left(h_{1}, Y\right), h \in c(g, X), \bar{h} \in c(\bar{g}, X) \\
g X_{\bar{g}, \bar{h}} \cap X_{g, h} \neq \emptyset
\end{array}
\end{array}\right\} .
$$

Indeed, one can define a bijection as follows: $g_{s} \mapsto g, g_{t} \mapsto \bar{g}, h \mapsto h, \bar{h} \mapsto \bar{h}$. Then we have the following.

$$
\begin{aligned}
\text { RHS of (4) }= & \sum_{\substack{h, \bar{h} \\
h \bar{h}=h_{1}}} \sum_{s, t} \theta\left(g_{s}, g_{t}\right)\left(\left.\pi(\xi)\right|_{\left.g_{s} X_{g_{t}, \bar{h}} \cap X_{g_{s}, h}\right)}\right. \\
= & \sum_{j} \sum_{\substack{g, \bar{g} \\
g \bar{g}=g_{j}}} \sum_{\substack{h \in c\left(\bar{h}, h \bar{h}=h_{1} \\
h \in c, X\right), \bar{h} \in c(\bar{g}, X)}} \theta(g, \bar{g})\left(\left.\pi(\xi)\right|_{\left.g X_{\bar{g}, \bar{h}} \cap X_{g, h}\right)}\right. \\
& \text { (use the above bijection to do change of variables) } \\
= & \sum_{j} \sum_{\substack{g, \bar{g} \\
g \bar{g}=g_{j}}} \theta(g, \bar{g})\left(\left.\pi(\xi)\right|_{X_{j}}\right)=\text { LHS of (4). }
\end{aligned}
$$

The 2nd last equality holds since for every $g, \bar{g}$ with $g \bar{g}=g_{j}$,

$$
X_{j}=\bigsqcup_{\substack{h, \bar{h}, h \bar{h}=h_{1} \\ h \in c(g, X), \bar{h} \in c(\bar{g}, X)}}\left(g X_{\bar{g}, \bar{h}} \cap X_{g, h}\right),
$$

which can be checked easily.

To check (5), observe for every $j$,

$$
\begin{aligned}
& \sum_{h_{2}, k} \theta\left(g_{j}, g_{k}\right)\left(\left.\pi(\xi)\right|_{g_{j} X_{k} \cap X_{j}}\right) \\
= & \sum_{h_{2}, k} \theta\left(g_{j}, g_{k}\right)\left(\left.\pi(\xi)\right|_{g_{j} X_{g_{k}, h_{2}} \cap X_{j}}\right) \text { (as } X_{k}=X_{g_{k}, h_{2}} \text { by convention) } \\
= & \sum_{g} \sum_{\substack{h_{2} \\
h_{2} \in c(g, X)}} \theta\left(g_{j}, g\right)\left(\left.\pi(\xi)\right|_{g_{j} X_{g, h_{2}} \cap X_{j}}\right) \\
= & \sum_{g} \theta\left(g_{j}, g\right)\left(\left.\pi(\xi)\right|_{X_{j}}\right) .
\end{aligned}
$$

The last equality holds since $X=\sqcup_{h_{2}}$ s.t. $h_{2} \in c(g, X) g_{j} X_{g, h_{2}}$ for every $g$.

Step 2: $S_{1}$ is an isomorphism. 
Recall $L: N_{0}(G, X) \rightarrow N_{0}(H, Y)$ is the inverse of $\pi$. Then we may define a map $T_{1}: C_{f}\left(H, N_{0}(H, Y)^{*}\right) \rightarrow C_{f}\left(G, N_{0}(G, X)^{*}\right)$ by setting $T_{1}\left(f^{\prime}\right)=f^{\prime \prime}$, where

$$
f^{\prime \prime}\left(g_{1}\right)(\eta):=\sum_{j} f^{\prime}\left(h_{j}\right)\left(\left.L(\eta)\right|_{Y_{j}}\right) .
$$

Here $\eta \in N_{0}(G, X)$ and $Y_{j}:=\left\{y \in Y: c^{\prime}\left(h_{j}^{-1}, y\right)=g_{1}^{-1}\right\}$.

Let $f \in C_{f}\left(G, N_{0}(G, X)^{*}\right)$, write $f^{\prime}=S_{1} f, f^{\prime \prime}=T_{1} f^{\prime}$. We check $T_{1} S_{1}=i d$. Equivalently, $f^{\prime \prime}=f$. A calculation shows

$$
\begin{aligned}
& f^{\prime \prime}\left(g_{1}\right)(\eta) \\
= & \sum_{j} f^{\prime}\left(h_{j}\right)\left(\left.L(\eta)\right|_{Y_{j}}\right)\left(\text { def. of } T_{1}\right) \\
= & \sum_{j} \sum_{i} f\left(g_{i}\right)\left(\left.\pi\left(\left.L(\eta)\right|_{Y_{j}}\right)\right|_{X_{g_{i}, h_{j}}}\right)\left(\text { def. of } S_{1}\right) \\
= & \sum_{j, i} f\left(g_{i}\right)\left(\left.\eta\right|_{X_{g_{i}, h_{j}} \cap \psi\left(Y_{j}\right)}\right) .(\text { Lemma 3.4) }
\end{aligned}
$$

Recall by our notation, $X_{g_{i}, h_{j}}:=\left\{x \in X: c\left(g_{i}^{-1}, x\right)=h_{j}^{-1}\right\}$. Now, it is easy to check that $X_{g_{i}, h_{j}} \cap \psi\left(Y_{j}\right)=\emptyset$ unless $g_{i}=g_{1}$. And $X_{g_{1}, h_{j}} \cap \psi\left(Y_{j}\right)=X_{g_{1}, h_{j}}$ by Lemma 3.1.

Hence, $f^{\prime \prime}\left(g_{1}\right)(\eta)=\sum_{j} f\left(g_{1}\right)\left(\left.\eta\right|_{X_{g_{1}, h_{j}}}\right)=f\left(g_{1}\right)(\eta)$.

So $T_{1} S_{1}=i d$. By symmetry, $S_{1} T_{1}=i d$.

4.3. General case. Based on the definition of $S_{i}$ for $i=0,1$, it is natural to consider the following general formula for $S_{n}$.

For all $n \geq 2$, let $\theta \in C_{f}\left(G^{n}, N_{0}(G, X)^{*}\right)$, define $S_{n}(\theta)=\theta^{\prime} \in C_{f}\left(H^{n}, N_{0}(H, Y)^{*}\right)$ by setting for every $\xi \in N_{0}(H, Y)$,

$$
\theta^{\prime}\left(h_{0}, \ldots, h_{n-1}\right)(\xi):=\sum_{t_{0}, \ldots, t_{n-1}} \theta\left(g_{t_{0}}, \ldots, g_{t_{n-1}}\right)\left(\left.\pi(\xi)\right|_{\left[t_{0}, \ldots, t_{n-1}\right]}\right) .
$$

Here, $c^{\prime}\left(h_{i}^{-1}, Y\right)=\left\{g_{t_{i}}^{-1}: t_{i} \in T_{i}\right\}$ for some finite set $T_{i}$ for all $i=0, \cdots, n-1$,

$$
\begin{aligned}
X_{t_{i}} & :=\left\{x: c\left(g_{t_{i}}^{-1}, x\right)=h_{i}^{-1}\right\} \\
{\left[t_{0}, \ldots, t_{n-1}\right] } & :=X_{t_{0}} \cap g_{t_{0}} X_{t_{1}} \cap \cdots \cap\left(g_{t_{0}} \cdots g_{t_{n-2}}\right) X_{t_{n-1}} .
\end{aligned}
$$

Strictly speaking, the index set for the above $\sum$ in (6) should be $\left(g_{t_{0}}, \ldots, g_{t_{n-1}}\right)$, but we simplify it to $\left(t_{0}, \ldots, t_{n-1}\right)$ for convenience. Again, we will switch to the notation $X_{g_{t_{i}}, h_{i}}$ once using $X_{t_{i}}$ causes confusion. We also reserve the notation $g_{i}$ for an arbitrary element in $G$.

Clearly, $S^{n}$ is well-defined. Indeed, observe that

$$
\operatorname{supp}\left(\theta^{\prime}\right) \subseteq \bigcup_{\left(g_{t_{0}}, \cdots, g_{t_{n-1}}\right) \in \operatorname{supp}(\theta)} c\left(g_{t_{0}}, X\right) \times \cdots \times c\left(g_{t_{n-1}}, X\right) .
$$

Hence, $\operatorname{supp}(\theta)$ is finite implies $\operatorname{supp}\left(\theta^{\prime}\right)$ is finite.

Our goal is to check that $\partial_{n} S_{n}=S_{n-1} \partial_{n}$ holds and $S_{n}$ is a bijection for each $n \geq 2$. This will finish the proof of Theorem 1.1 . 
Step 1: We claim $\partial_{n} S_{n}=S_{n-1} \partial_{n}$.

Take any $\theta \in C_{f}\left(G^{n}, N_{0}(G, X)^{*}\right)$, write $\theta^{\prime}=S_{n}(\theta)$ and $f=\partial_{n} \theta$. Fix any $\left(h_{1}, \ldots, h_{n-1}\right) \in H^{n-1}$ and any $\xi \in N_{0}(H, Y)$.

Now, we calculate both $\left[\partial_{n} S_{n}(\theta)\right]\left(h_{1}, \ldots, h_{n-1}\right)(\xi)$ and $\left[S_{n-1} \partial_{n}(\theta)\right]\left(h_{1}, \ldots, h_{n-1}\right)(\xi)$ and show they are equal.

First, we have the following calculation.

$$
\begin{aligned}
& {\left[\partial_{n} S_{n}(\theta)\right]\left(h_{1}, \ldots, h_{n-1}\right) } \\
= & {\left[\partial_{n} \theta^{\prime}\right]\left(h_{1}, \ldots, h_{n-1}\right) } \\
= & \sum_{h_{0} \in H} h_{0}^{-1} \theta^{\prime}\left(h_{0}, \ldots, h_{n-1}\right)+\sum_{i=1}^{n-1}(-1)^{i} \sum_{\substack{h, \bar{h} \in H \\
h \bar{h}=h_{i}}} \theta^{\prime}\left(h_{1}, \ldots, h_{i-1}, h, \bar{h}, h_{i+1}, \ldots, h_{n-1}\right) \\
& +(-1)^{n} \sum_{h_{n} \in H} \theta^{\prime}\left(h_{1}, \ldots, h_{n}\right) .
\end{aligned}
$$

Evaluate both sides of the above at the element $\xi$, then plug in the definition of $\theta^{\prime}$, i.e. (6) into the RHS of the above, we deduce that

$$
\begin{aligned}
& {\left[\partial_{n} S_{n}(\theta)\right]\left(h_{1}, \ldots, h_{n-1}\right)(\xi) } \\
= & \sum_{h_{0} \in H} \sum_{t_{0}, \ldots, t_{n-1}}\left\langle\theta\left(g_{t_{0}}, \ldots, g_{t_{n-1}}\right),\left.\pi\left(h_{0} \xi\right)\right|_{\left[t_{0}, \ldots, t_{n-1}\right]}\right\rangle \\
& +\sum_{i=1}^{n-1}(-1)^{i} \sum_{\substack{h, \bar{h} \in H \\
h \bar{h}=h_{i}}} \sum_{\substack{t_{1}, \ldots, t_{i-1}, s \\
k, t_{i+1}, \ldots, t_{n-1}}}\left\langle\theta\left(g_{t_{1}}, \ldots, g_{t_{i-1}}, g_{s}, g_{k}, g_{t_{i+1}}, \ldots, g_{t_{n-1}}\right),\right. \\
& +(-1)^{n} \sum_{h_{n} \in H} \sum_{t_{1}, \ldots, t_{n}}\left\langle\left.\theta(\xi)\right|_{\left[t_{1}, \ldots, t_{i-1}, s, k, t_{i+1}, \ldots, t_{n-1}\right]}\right\rangle
\end{aligned}
$$

Here, we use $\langle-,-\rangle$ to denote the evaluation, i.e. evaluating the first entry (a function) at the second entry (a variable). Let us recall some notations used here:

$$
X_{s}:=\left\{x: c\left(g_{s}^{-1}, x\right)=h^{-1}\right\}, \quad X_{k}:=\left\{x: c\left(g_{k}^{-1}, x\right)=\bar{h}^{-1}\right\} .
$$

$$
\begin{aligned}
& {\left[t_{1}, \ldots, t_{i-1}, s, k, t_{i+1}, \ldots, t_{n-1}\right]:=} \\
& X_{t_{1}} \cap g_{t_{1}} X_{t_{2}} \cap \cdots \cap\left(g_{t_{1}} \cdots g_{t_{i-2}}\right) X_{t_{i-1}} \cap\left(g_{t_{1}} \cdots g_{t_{i-1}}\right) X_{s} \\
& \quad \cap\left(g_{t_{1}} \cdots g_{t_{i-1}} g_{s}\right) X_{k} \cap \cdots \cap\left(g_{t_{1}} \cdots g_{t_{i-1}} g_{s} g_{k} g_{t_{i+1}} \cdots g_{t_{n-2}}\right) X_{t_{n-1}}
\end{aligned}
$$

Second, another calculation tells us the following.

$$
\begin{aligned}
& {\left[S_{n-1} \partial_{n} \theta\right]\left(h_{1}, \ldots, h_{n-1}\right)(\xi) } \\
= & {\left[S_{n-1} f\right]\left(h_{1}, \ldots, h_{n-1}\right)(\xi) } \\
= & \sum_{t_{1}, \ldots, t_{n-1}} f\left(g_{t_{1}}, \ldots, g_{t_{n-1}}\right)\left(\left.\pi(\xi)\right|_{\left[t_{1}, \ldots, t_{n-1}\right]}\right) . \quad\left(\text { def. of } S_{n-1}\right)
\end{aligned}
$$


Now, recall that $f=\partial_{n} \theta$ and the definition of $\partial_{n}$ from Section 2, we get

$$
\begin{aligned}
f\left(g_{t_{1}}, \ldots, g_{t_{n-1}}\right)= & \sum_{g_{0} \in G} g_{0}^{-1} \theta\left(g_{0}, g_{t_{1}}, \ldots, g_{t_{n-1}}\right) \\
& +\sum_{i=1}^{n-1}(-1)^{i} \sum_{\substack{g, \bar{g} \in G \\
g \bar{g}=g_{t_{i}}}} \theta\left(g_{t_{1}}, \ldots, g_{t_{i-1}}, g, \bar{g}, g_{t_{i+1}}, \ldots, g_{t_{n-1}}\right) \\
& +(-1)^{n} \sum_{g_{n} \in G} \theta\left(g_{t_{1}}, \ldots, g_{t_{n-1}}, g_{n}\right) .
\end{aligned}
$$

Evaluate both sides of the above at $\left.\pi(\xi)\right|_{\left[t_{1}, \ldots, t_{n-1}\right]}$ and combine with (10), we get

$$
\begin{aligned}
& {\left[S_{n-1} \partial_{n} \theta\right]\left(h_{1}, \ldots, h_{n-1}\right)(\xi) } \\
= & \sum_{t_{1}, \ldots, t_{n-1}} \sum_{g_{0} \in G}\left\langle g_{0}^{-1} \theta\left(g_{0}, g_{t_{1}}, \ldots, g_{t_{n-1}}\right),\left.\pi(\xi)\right|_{\left[t_{1}, \ldots, t_{n-1}\right]}\right\rangle \\
& +\sum_{t_{1}, \ldots, t_{n-1}} \sum_{i=1}^{n-1}(-1)^{i} \sum_{\substack{g, \bar{g} \in G \\
g \bar{g}=g_{t_{i}}}}\left\langle\theta\left(g_{t_{1}}, \ldots, g_{t_{i-1}}, g, \bar{g}, g_{t_{i+1}}, \ldots, g_{t_{n-1}}\right),\left.\pi(\xi)\right|_{\left[t_{1}, \ldots, t_{n-1}\right]}\right\rangle \\
& +\sum_{t_{1}, \ldots, t_{n-1}}(-1)^{n} \sum_{g_{n} \in G}\left\langle\theta\left(g_{t_{1}}, \ldots, g_{t_{n-1}}, g_{n}\right),\left.\pi(\xi)\right|_{\left[t_{1}, \ldots, t_{n-1}\right]}\right\rangle .
\end{aligned}
$$

We aim to show $(8)=(11)$. To do this, we just need to compare all the corresponding summands and prove they are equal, i.e. it suffices to check the following hold.

(a)

$$
\begin{aligned}
\sum_{h_{0} \in H} \sum_{t_{0}, \ldots, t_{n-1}}\left\langle\theta\left(g_{t_{0}}, \ldots, g_{t_{n-1}}\right),\left.\pi\left(h_{0} \xi\right)\right|_{\left[t_{0}, \ldots, t_{n-1}\right]}\right\rangle & \\
& =\sum_{t_{1}, \ldots, t_{n-1}} \sum_{g_{0} \in G}\left\langle g_{0}^{-1} \theta\left(g_{0}, g_{t_{1}}, \ldots, g_{t_{n-1}}\right),\left.\pi(\xi)\right|_{\left[t_{1}, \ldots, t_{n-1}\right]}\right\rangle .
\end{aligned}
$$

(b) For any $1 \leq i \leq n-1$,

$$
\begin{array}{r}
\sum_{\substack{h, \bar{h} \in H \\
h \bar{h}=h_{i}}} \sum_{\substack{t_{1}, \ldots, t_{i-1}, s \\
k, t_{i+1}, \ldots, t_{n-1}}}\left\langle\theta\left(g_{t_{1}}, \ldots, g_{t_{i-1}}, g_{s}, g_{k}, g_{t_{i+1}}, \ldots, g_{t_{n-1}}\right),\left.\pi(\xi)\right|_{\left[t_{1}, \ldots, t_{i-1}, s, k, t_{i+1}, \ldots, t_{n-1}\right]}\right\rangle \\
\quad=\sum_{t_{1}, \ldots, t_{n-1}} \sum_{\substack{g, \bar{g} \in G \\
g \bar{g}=g_{t_{i}}}}\left\langle\theta\left(g_{t_{1}}, \ldots, g_{t_{i-1}}, g, \bar{g}, g_{t_{i+1}}, \ldots, g_{t_{n-1}}\right),\left.\pi(\xi)\right|_{\left[t_{1}, \ldots, t_{n-1}\right]}\right\rangle .
\end{array}
$$

(c)

$$
\begin{aligned}
\sum_{h_{n} \in H} \sum_{t_{1}, \ldots, t_{n}}\left\langle\theta\left(g_{t_{1}}, \ldots, g_{t_{n}}\right),\left.\pi(\xi)\right|_{\left[t_{1}, \ldots, t_{n}\right]}\right\rangle & \\
& =\sum_{t_{1}, \ldots, t_{n-1}} \sum_{g_{n} \in G}\left\langle\theta\left(g_{t_{1}}, \ldots, g_{t_{n-1}}, g_{n}\right),\left.\pi(\xi)\right|_{\left[t_{1}, \ldots, t_{n-1}\right]}\right\rangle .
\end{aligned}
$$


We prove (a), (b), (c) below respectively.

Proof of (a). By Lemma 3.3, $\left.\pi\left(h_{0} \xi\right)\right|_{\left[t_{0}, \ldots, t_{n-1}\right]}=g_{t_{0}}\left(\left.\pi(\xi)\right|_{g_{t_{0}}^{-1}\left[t_{0}, \ldots, t_{n-1}\right]}\right)$. Then,

Top expression in (a)

$$
\begin{aligned}
& =\sum_{h_{0} \in H} \sum_{t_{0}, \ldots, t_{n-1}}\left\langle\theta\left(g_{t_{0}}, \ldots, g_{t_{n-1}}\right),\left.\pi\left(h_{0} \xi\right)\right|_{\left[t_{0}, \ldots, t_{n-1}\right]}\right\rangle \\
& =\sum_{h_{0} \in H} \sum_{t_{0}, \ldots, t_{n-1}}\left\langle\theta\left(g_{t_{0}}, \ldots, g_{t_{n-1}}\right), g_{t_{0}}\left(\left.\pi(\xi)\right|_{g_{t_{0}}^{-1}\left[t_{0}, \ldots, t_{n-1}\right]}\right)\right\rangle \\
& =\sum_{h_{0} \in H} \sum_{t_{0}, \ldots, t_{n-1}}\left\langle g_{t_{0}}^{-1} \theta\left(g_{t_{0}}, \ldots, g_{t_{n-1}}\right),\left.\pi(\xi)\right|_{g_{t_{0}}^{-1} X_{t_{0}} \cap\left[t_{1}, \ldots, t_{n-1}\right]}\right\rangle
\end{aligned}
$$

(def. of dual module and by $(7), g_{t_{0}}^{-1}\left[t_{0}, \ldots, t_{n-1}\right]=g_{t_{0}}^{-1} X_{t_{0}} \cap\left[t_{1}, \ldots, t_{n-1}\right]$ )

$$
=\sum_{t_{1}, \ldots, t_{n-1}} \sum_{h_{0} \in H} \sum_{t_{0}}\left\langle g_{t_{0}}^{-1} \theta\left(g_{t_{0}}, \ldots, g_{t_{n-1}}\right),\left.\pi(\xi)\right|_{g_{t_{0}}^{-1} X_{t_{0}} \cap\left[t_{1}, \ldots, t_{n-1}\right]}\right\rangle
$$

(switching sums is possible as $t_{1}, \ldots, t_{n-1}$ and $h_{0}$ are independent)

$$
=\sum_{t_{1}, \ldots, t_{n-1}} \sum_{h_{0} \in H} \sum_{\substack{g_{t_{0}} \\ g_{t_{0}} \in c^{\prime}\left(h_{0}, Y\right)}}\left\langle g_{t_{0}}^{-1} \theta\left(g_{t_{0}}, \ldots, g_{t_{n-1}}\right),\left.\pi(\xi)\right|_{g_{t_{0}}^{-1} X_{g_{t_{0}}, h_{0}} \cap\left[t_{1}, \ldots, t_{n-1}\right]}\right\rangle
$$

(notation convention, check the explanation given when defing $S_{n}$, i.e. (6))

$$
=\sum_{t_{1}, \ldots, t_{n-1}} \sum_{g_{0} \in G} \sum_{h_{0} \in c\left(g_{0}, X\right)}\left\langle g_{0}^{-1} \theta\left(g_{0}, \ldots, g_{t_{n-1}}\right),\left.\pi(\xi)\right|_{g_{0}^{-1} X_{g_{0}, h_{0}} \cap\left[t_{1}, \ldots, t_{n-1}\right]}\right\rangle .
$$

The last equality holds for the following reason:

The map $h_{0} \mapsto h_{0}, g_{t_{0}} \mapsto g_{0}$ induces a bijection between the two index sets: $\left\{\left(h_{0}, g_{t_{0}}\right): h_{0} \in H, g_{t_{0}} \in c^{\prime}\left(h_{0}, Y\right)\right\}$ and $\left\{\left(g_{0}, h_{0}\right): g_{0} \in G, h_{0} \in c\left(g_{0}, X\right)\right\}$. This explains the change of the index sets under the two rightmost sums. So under the above bijection, $g_{t_{0}}^{-1} X_{g_{t_{0}}, h_{0}}$ is replaced by $g_{0}^{-1} X_{g_{0}, h_{0}}$.

Now, recall $x \in g_{0}^{-1} X_{g_{0}, h_{0}}$ iff $g_{0} x \in X_{g_{0}, h_{0}}$ iff $c\left(g_{0}, x\right)=h_{0}$. Therefore, for each $g_{0} \in G, X=\sqcup_{h_{0} \in c\left(g_{0}, X\right)} g_{0}^{-1} X_{g_{0}, h_{0}}$.

Using this fact, we can continue to simplify (12) to the following expressions.

The top expression in (a)

$$
\begin{aligned}
& =\sum_{t_{1}, \ldots, t_{n-1}} \sum_{g_{0} \in G}\left\langle g_{0}^{-1} \theta\left(g_{0}, \ldots, g_{t_{n-1}}\right),\left.\pi(\xi)\right|_{\left(\sqcup_{h_{0} \in c\left(g_{0}, X\right)} g_{0}^{-1} X_{g_{0}, h_{0}}\right) \cap\left[t_{1}, \ldots, t_{n-1}\right]}\right\rangle \\
& =\sum_{t_{1}, \ldots, t_{n-1}} \sum_{g_{0} \in G}\left\langle g_{0}^{-1} \theta\left(g_{0}, \ldots, g_{t_{n-1}}\right),\left.\pi(\xi)\right|_{X \cap\left[t_{1}, \ldots, t_{n-1}\right]}\right\rangle \\
& =\sum_{t_{1}, \ldots, t_{n-1}} \sum_{g_{0} \in G}\left\langle g_{0}^{-1} \theta\left(g_{0}, \ldots, g_{t_{n-1}}\right),\left.\pi(\xi)\right|_{\left[t_{1}, \ldots, t_{n-1}\right]}\right\rangle \\
& =\text { The bottom expression in (a). }
\end{aligned}
$$

Proof of (b). The proof relies on the following two facts: 
(Fact 1) For every fixed $t_{1}, \ldots, t_{i-1}, t_{i+1}, \ldots, t_{n-1}$, the map $t_{j} \mapsto t_{j}(\forall j \neq i)$, $s \mapsto g, k \mapsto \bar{g}$ induces a bijection between the index sets:

$$
\left\{\begin{array}{l|l}
\left(t_{1}, \ldots, t_{i-1}, t_{i+1}, \ldots, t_{n-1}, s, k\right) & \begin{array}{l}
h \bar{h}=h_{i} \\
g_{s} \in c^{\prime}(h, Y), g_{k} \in c^{\prime}(\bar{h}, Y) \\
{\left[t_{1}, \ldots, t_{i-1}, s, k, t_{i+1}, \ldots, t_{n-1}\right] \neq \emptyset}
\end{array}
\end{array}\right\}
$$

and

$$
\left\{\begin{array}{l|l}
\left(t_{1}, \ldots, t_{n-1}, g, \bar{g}\right) & \begin{array}{l}
g \bar{g}=g_{t_{i}}, h \bar{h}=h_{i} \\
h \in c(g, X), \bar{h} \in c(\bar{g}, X), g_{t_{i}} \in c^{\prime}\left(h_{i}, Y\right) \\
{\left[t_{1}, \ldots, t_{i-1}, g, \bar{g}, t_{i+1}, \ldots, t_{n-1}\right] \neq \emptyset}
\end{array}
\end{array}\right\}
$$

Indeed, just remember that the index $s$ stands for $g_{s}, k$ stands for $g_{k}, t_{j}$ stands for $g_{t_{j}}$ and $g_{t_{i}}$ is determined by $g$ and $\bar{g}$.

Here, $\left[t_{1}, \ldots, t_{i-1}, g, \bar{g}, t_{i+1}, \ldots, t_{n-1}\right]$ is the image of $\left[t_{1}, \ldots, t_{i-1}, s, k, t_{i+1}, \ldots, t_{n-1}\right]$ under the bijection as in Fact 1. More precisely, from the def. (9), we know

$$
\begin{aligned}
& {\left[t_{1}, \ldots, t_{i-1}, g, \bar{g}, t_{i+1}, \ldots, t_{n-1}\right]=X_{t_{1}} \cap g_{t_{1}} X_{t_{2}} \cap \cdots \cap\left(g_{t_{1}} \cdots g_{t_{i-2}}\right) X_{t_{i-1}}} \\
& \cap\left(g_{t_{1}} \cdots g_{t_{i-1}}\right) X_{g} \cap\left(g_{t_{1}} \cdots g_{t_{i-1}} g\right) X_{\bar{g}} \cap\left(g_{t_{1}} \cdots g_{t_{i-1}} g \bar{g}\right) X_{t_{i+1}} \\
& \quad \cap\left(g_{t_{1}} \cdots g_{t_{i-1}} g \bar{g} g_{t_{i+1}}\right) X_{t_{i+2}} \cap \cdots \cap\left(g_{t_{1}} \cdots g_{t_{i-1}} g \bar{g} g_{t_{i+1}} \cdots g_{t_{n-2}}\right) X_{t_{n-1}}
\end{aligned}
$$

We remind the reader in the above expression, $X_{g}=X_{g, h}=\left\{x: c\left(g^{-1}, x\right)=h^{-1}\right\}$ and $X_{\bar{g}}=\left\{x: c\left(\bar{g}^{-1}, x\right)=\bar{h}^{-1}\right\}$.

(Fact 2) For all $g, \bar{g} \in G$ satisfying $g \bar{g}=g_{t_{i}}$, we have

$$
\begin{aligned}
& \quad \bigsqcup_{\substack{h, \bar{h}, h \bar{h}=h_{i} \\
h \in c(g, X), \bar{h} \in c(\bar{g}, X)}}\left(g_{t_{1}} \cdots g_{t_{i-1}}\right) X_{g, h} \cap\left(g_{t_{1}} \cdots g_{t_{i-1}} g\right) X_{\bar{g}, \bar{h}} \cap\left(g_{t_{1}} \cdots g_{t_{i-1}} g \bar{g}\right) X_{t_{i+1}} \\
& \quad=\left(g_{t_{1}} \cdots g_{t_{i-1}}\right) X_{t_{i}} \cap\left(g_{t_{1}} \cdots g_{t_{i}}\right) X_{t_{i+1}} .
\end{aligned}
$$

Recall $X_{t_{i+1}}=X_{g_{t_{i+1}}, h_{i+1}}=\left\{x: c\left(g_{t_{i+1}}^{-1}, x\right)=h_{i+1}^{-1}\right\}, X_{g, h}=\left\{x: c\left(g^{-1}, x\right)=h^{-1}\right\}$ and $X_{\bar{g}, \bar{h}}=\left\{x: c\left(\bar{g}^{-1}, x\right)=\bar{h}^{-1}\right\}$. Then Fact 2 is easy to verify using cocycle identities.

Now, we can prove (b) as follows.

By Fact 1,

The top expression in (b)

$$
\begin{gathered}
=\sum_{t_{1}, \ldots, t_{n-1}} \sum_{\substack{g, \bar{g} \in G \\
g \bar{g}=g_{t_{i}}}} \sum_{\substack{h, \bar{h}, h \bar{h}=h_{i} \\
h \in c(g, X), \bar{h} \in c(\bar{g}, X)}}\left\langle\theta\left(g_{t_{1}}, \ldots, g_{t_{i-1}}, g, \bar{g}, g_{t_{i+1}}, \ldots, g_{t_{n-1}}\right),\right. \\
\left.\left.\pi(\xi)\right|_{\left[t_{1}, \ldots, t_{i-1}, g, \bar{g}, t_{i+1}, \ldots, t_{n-1}\right]}\right\rangle .
\end{gathered}
$$


Since $g \bar{g}=g_{t_{i}}$, we can rewrite (13) as

$$
\begin{gathered}
{\left[t_{1}, \ldots, t_{i-1}, g, \bar{g}, t_{i+1}, \ldots, t_{n-1}\right]=X_{t_{1}} \cap g_{t_{1}} X_{t_{2}} \cap \cdots \cap\left(g_{t_{1}} \cdots g_{t_{i-2}}\right) X_{t_{i-1}}} \\
\cap \frac{\left(g_{t_{1}} \cdots g_{t_{i-1}}\right) X_{g, h} \cap\left(g_{t_{1}} \cdots g_{t_{i-1}} g\right) X_{\bar{g}, \bar{h}} \cap\left(g_{t_{1}} \cdots g_{t_{i-1}} g \bar{g}\right) X_{t_{i+1}}}{\cap\left(g_{t_{1}} \cdots g_{t_{i-1}} g_{t_{i}} g_{t_{i+1}}\right) X_{t_{i+2}} \cap \cdots \cap\left(g_{t_{1}} \cdots g_{t_{i-1}} g_{t_{i}} g_{t_{i+1}} \cdots g_{t_{n-2}}\right) X_{t_{n-1}}} .
\end{gathered}
$$

Now, observe that the underlined part above is exactly the one appeared in Fact 2 , and the rest pieces in (15) are exactly the ones appeared in the definition of $\left[t_{1}, \ldots, t_{n-1}\right]$, which is recalled below

$$
\begin{aligned}
{\left[t_{1}, \ldots, t_{n-1}\right]=} & X_{t_{1}} \cap g_{t_{1}} X_{t_{2}} \cap \cdots \cap\left(g_{t_{1}} \cdots g_{t_{i-2}}\right) X_{t_{i-1}} \\
& \cap \frac{\left(g_{t_{1}} \cdots g_{t_{i-1}}\right) X_{t_{i}} \cap\left(g_{t_{1}} \cdots g_{t_{i}}\right) X_{t_{i+1}}}{\cap\left(g_{t_{1}} \cdots g_{t_{i-1}} g_{t_{i}} g_{t_{i+1}}\right) X_{t_{i+2}} \cap \cdots \cap\left(g_{t_{1}} \cdots g_{t_{i-1}} g_{t_{i}} g_{t_{i+1}} \cdots g_{t_{n-2}}\right) X_{t_{n-1}} .}
\end{aligned}
$$

Apply the above observation, we can continue the computation in (14) as follows.

First, we can move

$$
\sum_{\substack{h, \bar{h}, h \bar{h}=h_{i} \\ h \in c(g, X), \bar{h} \in c(\bar{g}, X)}}
$$

inside $\langle-,-\rangle$ and put it before the 2nd entry, i.e. $\left.\pi(\xi)\right|_{\left[t_{1}, \ldots, t_{i-1}, g, \bar{g}, t_{i+1}, \ldots, t_{n-1}\right]}$, to get

$$
\left.\sum_{\substack{h, \bar{h}, h \bar{h}=h_{i} \\ h \in c(g, X), \bar{h} \in c(\bar{g}, X)}} \pi(\xi)\right|_{\left[t_{1}, \ldots, t_{i-1}, g, \bar{g}, t_{i+1}, \ldots, t_{n-1}\right]}=\left.\pi(\xi)\right|_{\mathcal{\jmath}} .
$$

Here,

$$
\begin{aligned}
\mathcal{J} & :=\bigsqcup_{\substack{h, \bar{h}, h \bar{h}=h_{i} \\
h \in c(g, X), \bar{h} \in c(\bar{g}, X)}}\left[t_{1}, \ldots, t_{i-1}, g, \bar{g}, t_{i+1}, \ldots, t_{n-1}\right] \\
& \left.=\left[t_{1}, \ldots, t_{n-1}\right] . \quad \text { (by Fact } 2+(15)\right)
\end{aligned}
$$

Put all the above information together, we finally arrive at the following.

The top expression in (b)

$$
\begin{aligned}
& =\sum_{t_{1}, \ldots, t_{n-1}} \sum_{\substack{g, \bar{g} \in G \\
g \bar{g}=g_{t_{i}}}}\left\langle\theta\left(g_{t_{1}}, \ldots, g_{t_{i-1}}, g, \bar{g}, g_{t_{i+1}}, \ldots, g_{t_{n-1}}\right),\left.\pi(\xi)\right|_{\left[t_{1}, \ldots, t_{n-1}\right]}\right\rangle \\
& =\text { The bottom expression in (b). }
\end{aligned}
$$

This finishes the proof of (b).

Proof of (c).

The top expression in (c)

$$
=\sum_{t_{1}, \ldots, t_{n-1}} \sum_{h_{n} \in H} \sum_{t_{n}}\left\langle\theta\left(g_{t_{1}}, \ldots, g_{t_{n}}\right),\left.\pi(\xi)\right|_{\left[t_{1}, \ldots, t_{n}\right]}\right\rangle
$$




$$
\begin{aligned}
= & \sum_{t_{1}, \ldots, t_{n-1}} \sum_{h_{n} \in H} \sum_{t_{n}}\left\langle\theta\left(g_{t_{1}}, \ldots, g_{t_{n}}\right),\left.\pi(\xi)\right|_{\left[t_{1}, \ldots, t_{n-1}\right] \cap\left(g_{t_{1}} \cdots g_{t_{n-1}}\right) X_{t_{n}}}\right\rangle \\
& \left(\text { def. of }\left[t_{1}, \ldots, t_{n}\right]\right) \\
= & \sum_{t_{1}, \ldots, t_{n-1}} \sum_{g_{n} \in G} \sum_{h_{n} \in c\left(g_{n}, X\right)}\left\langle\theta\left(g_{t_{1}}, \ldots, g_{t_{n-1}}, g_{n}\right),\left.\pi(\xi)\right|_{\left[t_{1}, \ldots, t_{n-1}\right] \cap\left(g_{t_{1}} \cdots g_{t_{n-1}}\right) X_{g_{n}, h_{n}}}\right\rangle .
\end{aligned}
$$

The last equality holds for the following reason.

Recall here $X_{t_{n}}=X_{g_{t_{n}}, h_{n}}=\left\{x: c\left(g_{t_{n}}^{-1}, x\right)=h_{n}^{-1}\right\}$, so $X_{t_{n}}$ is replaced by $X_{g_{n}, h_{n}}$ under the bijective map $t_{n} \mapsto g_{n}, h_{n} \mapsto h_{n}$ between the index sets

$$
\left\{\left(h_{n}, t_{n}\right): t_{n} \in c^{\prime}\left(h_{n}, Y\right), h_{n} \in H\right\}
$$

and

$$
\left\{\left(g_{n}, h_{n}\right): h_{n} \in c\left(g_{n}, X\right), g_{n} \in G\right\}
$$

Then, since

$$
X=\bigsqcup_{h_{n} \in c\left(g_{n}, X\right)}\left(g_{t_{1}} \cdots g_{t_{n-1}}\right) X_{g_{n}, h_{n}}
$$

we can continue the computation of the above expression as follows.

The top expression in (c)

$$
\begin{aligned}
& =\sum_{t_{1}, \ldots, t_{n-1}} \sum_{g_{n} \in G}\left\langle\theta\left(g_{t_{1}}, \ldots, g_{t_{n-1}}, g_{n}\right),\left.\pi(\xi)\right|_{\left.\left[t_{1}, \ldots, t_{n-1}\right] \cap \sqcup_{h_{n} \in c\left(g_{n}, X\right)}\left(g_{t_{1}} \cdots g_{t_{n-1}}\right) X_{g_{n}, h_{n}}\right\rangle}\right\rangle \\
& =\sum_{t_{1}, \ldots, t_{n-1}} \sum_{g_{n} \in G}\left\langle\theta\left(g_{t_{1}}, \ldots, g_{t_{n-1}}, g_{n}\right),\left.\pi(\xi)\right|_{\left[t_{1}, \ldots, t_{n-1}\right]}\right\rangle \\
& =\text { The bottom expression in (c). }
\end{aligned}
$$

Step 2: $S_{n}$ is a bijection.

By symmetry, we define a (linear) map $T_{n}: C_{f}\left(H^{n}, N_{0}(H, Y)^{*}\right) \rightarrow C_{f}\left(G^{n}, N_{0}(G, X)^{*}\right)$ by setting $T_{n}\left(\theta^{\prime}\right)=\theta^{\prime \prime}$, where

$$
\theta^{\prime \prime}\left(g_{0}, \ldots, g_{n-1}\right)(\eta):=\sum_{s_{0}, \ldots, s_{n-1}} \theta^{\prime}\left(h_{s_{0}}, \ldots, h_{s_{n-1}}\right)\left(\left.L(\eta)\right|_{\left[s_{0}, \ldots, s_{n-1}\right]}\right)
$$

Here, $\eta \in N_{0}(G, X), Y_{s_{i}}:=\left\{y \in Y: c^{\prime}\left(h_{s_{i}}^{-1}, y\right)=g_{i}^{-1}\right\}$ and

$$
\left[s_{0}, \ldots, s_{n-1}\right]:=Y_{s_{0}} \cap h_{s_{0}} Y_{s_{1}} \cap \cdots \cap\left(h_{s_{0}} \cdots h_{s_{n-2}}\right) Y_{s_{n-1}} .
$$

If we can prove the following claim, then by symmetry, we also have $S_{n} T_{n}=i d$, and hence $S_{n}$ is a bijection.

Claim: $T_{n} S_{n}=i d$.

Proof of the claim. Take any $\theta \in C_{f}\left(G^{n}, N_{0}(G, X)^{*}\right),\left(g_{0}, \ldots, g_{n-1}\right) \in G^{n}$ and any $\eta \in N_{0}(G, X)$, let $\theta^{\prime}=S_{n}(\theta)$ and $\theta^{\prime \prime}=T_{n}\left(\theta^{\prime}\right)$.

We aim to show $\theta=\theta^{\prime \prime}$, i.e. $\theta^{\prime \prime}\left(g_{0}, \ldots, g_{n-1}\right)(\eta)=\theta\left(g_{0}, \ldots, g_{n-1}\right)(\eta)$. 
From the definitions of $S_{n}$ and $T_{n}$, i.e. (6) and (16), we deduce that

$$
\begin{aligned}
& \theta^{\prime \prime}\left(g_{0}, \ldots, g_{n-1}\right)(\eta) \\
= & \sum_{s_{0}, \ldots, s_{n-1}} \theta^{\prime}\left(h_{s_{0}}, \ldots, h_{s_{n-1}}\right)\left(\left.L(\eta)\right|_{\left[s_{0}, \ldots, s_{n-1}\right]}\right) \\
= & \sum_{s_{0}, \ldots, s_{n-1}} \sum_{t_{0}, \ldots, t_{n-1}} \theta\left(g_{t_{0}}, \ldots, g_{t_{n-1}}\right)\left(\left.\pi\left(\left.L(\eta)\right|_{\left[s_{0}, \ldots, s_{n-1}\right]}\right)\right|_{\left[t_{0}, \ldots, t_{n-1}\right]}\right) .
\end{aligned}
$$

Since there are many variables here, let us recall some notations used above.

$\left[s_{0}, \ldots, s_{n-1}\right]$ is defined as in (17), and def. (7) in our context is the following.

$$
\left[t_{0}, \ldots, t_{n-1}\right]=X_{g_{t_{0}}, h_{s_{0}}} \cap g_{t_{0}} X_{g_{t_{1}}, h_{s_{1}}} \cap \cdots \cap\left(g_{t_{0}} \ldots g_{t_{n-2}}\right) X_{g_{t_{n-1}} h_{s_{n-1}}},
$$

where $X_{g_{i}, h_{s_{i}}}=\left\{x: c\left(g_{t_{i}}^{-1}, x\right)=h_{s_{i}}^{-1}\right\}$.

To continue the computation, we need the following facts.

(Fact 3) $\left.\pi\left(\left.L(\eta)\right|_{\left[s_{0}, \ldots, s_{n-1}\right]}\right)\right|_{\left[t_{0}, \ldots, t_{n-1}\right]}=\left.\eta\right|_{\left[t_{0}, \ldots, t_{n-1}\right] \cap \psi\left(\left[s_{0}, \ldots, s_{n-1}\right]\right)}$.

This is clear by Lemma 3.4.

(Fact 4) $\left[t_{0}, \ldots, t_{n-1}\right] \cap \psi\left(\left[s_{0}, \ldots, s_{n-1}\right]\right)=\emptyset$ unless $g_{t_{i}}=g_{i}$ for all $0 \leq i \leq n-1$. When these conditions hold, the intersection equals

$$
X_{g_{0}, s_{0}} \cap g_{0} X_{g_{1}, s_{1}} \cap \cdots \cap\left(g_{0} \cdots g_{n-2}\right) X_{g_{n-1}, s_{n-1}},
$$

where $X_{g_{i}, s_{i}}=\left\{x: c\left(g_{i}^{-1}, x\right)=h_{s_{i}}^{-1}\right\}$.

This fact can be checked using (17), (19) and Lemma 3.1.

Now, we can continue the computation of (18) as follows.

$$
\begin{aligned}
& \theta^{\prime \prime}\left(g_{0}, \ldots, g_{n-1}\right)(\eta) \\
& \stackrel{\text { Fact }}{=} \sum_{s_{0}, \ldots, s_{n-1}} \sum_{t_{0}, \ldots, t_{n-1}} \theta\left(g_{t_{0}}, \ldots, g_{t_{n-1}}\right)\left(\left.\eta\right|_{\left[t_{0}, \ldots, t_{n-1}\right] \cap \psi\left(\left[s_{0}, \ldots, s_{n-1}\right]\right)}\right) \\
& \stackrel{\text { Fact }}{=} \sum_{s_{0}, \ldots, s_{n-1}} \theta\left(g_{0}, \ldots, g_{n-1}\right)\left(\left.\eta\right|_{X_{g_{0}, s_{0}} \cap g_{0} X_{g_{1}, s_{1}} \cap \cdots \cap\left(g_{0} \cdots g_{n-2}\right) X_{g_{n-1}, s_{n-1}}}\right) \\
& =\theta\left(g_{0}, \ldots, g_{n-1}\right)\left(\left.\eta\right|_{\bigsqcup_{s_{0}, \ldots, s_{n-1}}}\left(X_{\left.g_{0}, s_{0} \cap g_{0} X_{g_{1}, s_{1}} \cap \cdots \cap\left(g_{0} \cdots g_{n-2}\right) X_{g_{n-1}, s_{n-1}}\right)}\right)\right. \\
& =\theta\left(g_{0}, \ldots, g_{n-1}\right)(\eta) \text {. }
\end{aligned}
$$

The last equality holds as

$$
X=\bigsqcup_{s_{0}, \ldots, s_{n-1}}\left(X_{g_{0}, s_{0}} \cap g_{0} X_{g_{1}, s_{1}} \cap \cdots \cap\left(g_{0} \cdots g_{n-2}\right) X_{g_{n-1}, s_{n-1}}\right),
$$

which can be checked directly. 


\section{Proof of Theorem 1.2}

The idea for the proof is similar to the previous one: For each $n$, we will construct a (linear) map $S^{n}$ from $C_{b}\left(H^{n}, N_{0}(H, Y)^{* *}\right)$ to $C_{b}\left(G^{n}, N_{0}(G, X)^{* *}\right)$ and check directly $S^{n}$ is a cochain map, i.e. $\partial^{n-1} S^{n-1}=S^{n} \partial^{n-1}$ holds. Then, we use symmetry to find the inverse of $S^{n}$. Since the proof of general case uses cumbersome notations, we include the proof of the initial cases $(n=1,2,3)$ to illustrate the main ideas.

5.1. Case $n=1$. Recall that $C_{b}\left(H^{0}, N_{0}(H, Y)^{* *}\right)=N_{0}(H, Y)^{* *}$. We define $S^{0}=$ $\pi^{* *}$ and $S^{1}(f)=f^{\prime}$, where

$$
f^{\prime}(g)(\tau):=\sum_{i}\left\langle\pi^{* *}\left(f\left(h_{i}\right)\right), \tau_{i}\right\rangle .
$$

Here, $f \in C_{b}\left(H, N_{0}(H, Y)^{* *}\right), \tau \in N_{0}(G, X)^{*}$ and $\tau_{i}=\left.\tau\right|_{X_{i}}$. Moreover, we write $X_{i}=\left\{x \in X: c\left(g^{-1}, x\right)=h_{i}^{-1}\right\}$, where $c(g, X)=\left\{h_{i} \mid i\right\}$.

Step 1: check $S^{1}$ is well-defined, i.e. $\sup _{g \in G}\left\|f^{\prime}(g)\right\|<\infty$.

Since $\left\langle\pi^{* *}\left(f\left(h_{i}\right)\right), \tau_{i}\right\rangle=f\left(h_{i}\right)\left(\tau_{i} \pi\right)$, it suffices to show that $\sum_{i}\left\|\tau_{i}\right\| /\|\tau\|$ is bounded (and independent of the choice of $g,\left\{X_{i}\right\}$ and $\left.n:=\# c(g, X)\right)$. This is done by the following lemma.

Lemma 5.1. Let $\tau \in N_{0}(G, X)^{*}$ and $X=\sqcup_{i} X_{i}$ be a finite clopen partition. Then $\sum_{i}\left\|\tau_{i}\right\| \leq\|\tau\|$.

Proof. For any $\epsilon>0$, take $a_{i} \in N_{0}(G, X)$ such that $\left\|a_{i}\right\|=1$ and $\left\|\tau_{i}\right\| \leq\left|\tau_{i}\left(a_{i}\right)\right|+\epsilon / n$ for all $i$.

Then $\sum_{i}|| \tau_{i} \| \leq \sum_{i}\left|\tau_{i}\left(a_{i}\right)\right|+\epsilon=\sum_{i} \tau\left(\left.a_{i}\right|_{X_{i}} \cdot \lambda_{i}\right)+\epsilon=\tau\left(\sum_{i}\left(\left.a_{i}\right|_{X_{i}} \cdot \lambda_{i}\right)\right)+\epsilon$, where $\lambda_{i} \in\{ \pm 1\}$ is the sign of $\tau_{i}\left(a_{i}\right)$.

Observe that $\sum_{i}\left(\left.a_{i}\right|_{X_{i}} \cdot \lambda_{i}\right) \in N_{0}(G, X)$ and $\left\|\sum_{i}\left(\left.a_{i}\right|_{X_{i}} \cdot \lambda_{i}\right)\right\| \leq 1$. Hence, $\sum_{i}\left\|\tau_{i}\right\| \leq$ $\|\tau\|+\epsilon$ for all $\epsilon>0$. So, $\sum_{i}\left\|\tau_{i}\right\| \leq\|\tau\|$.

Step 2: check $\partial^{0} S^{0}=S^{1} \partial^{0}$.

Take any $\xi \in N_{0}(H, Y)^{* *}, g \in G$ and $\tau \in N_{0}(G, X)^{*}$. We do computation as follows.

$$
\begin{aligned}
\left(S^{1} \partial^{0} \xi\right)(g)(\tau) & =\sum_{i}\left\langle\pi^{* *}\left(\left(\partial^{0} \xi\right)\left(h_{i}\right)\right), \tau_{i}\right\rangle \quad\left(\text { def. of } S^{1}\right) \\
& =\sum_{i}\left\langle\left(\partial^{0} \xi\right)\left(h_{i}\right), \tau_{i} \pi\right\rangle \quad\left(\text { def. of } \pi^{* *}\right) \\
& =\sum_{i}\left\langle h_{i} \xi, \tau_{i} \pi\right\rangle \quad\left(\text { def. of } \partial^{0}\right) \\
& =\sum_{i}\left\langle\xi, h_{i}^{-1}\left(\tau_{i} \pi\right)\right\rangle . \quad \text { (def. of dual action) }
\end{aligned}
$$




$$
\begin{aligned}
\left(\partial^{0} S^{0} \xi\right)(g)(\tau) & =\left[g\left(S^{0} \xi\right)\right](\tau) & & \left(\text { def. of } \partial^{0}\right) \\
& =\left(S^{0} \xi\right)\left(g^{-1} \tau\right) & & (\text { def. of dual action }) \\
& =\left\langle\pi^{* *} \xi, g^{-1} \tau\right\rangle & & \left(S^{0}=\pi^{* *}\right) \\
& =\left\langle\xi,\left(g^{-1} \tau\right) \pi\right\rangle & & \left(\text { def. of } \pi^{* *}\right) \\
& =\sum_{i}\left\langle\xi,\left(g^{-1} \tau_{i}\right) \pi\right\rangle . & & \left(\tau=\sum_{i} \tau_{i}\right)
\end{aligned}
$$

Therefore, to show the above two equations are equal, it suffices to check that for each $i$, we have $h_{i}^{-1}\left(\tau_{i} \pi\right)=\left(g^{-1} \tau_{i}\right) \pi$. Equivalently, we need to check that $\tau\left(\left.\pi\left(h_{i} \xi\right)\right|_{X_{i}}\right)=$ $\tau\left(\left.[g(\pi(\xi))]\right|_{X_{i}}\right)$ for each $\xi \in N_{0}(H, Y)$, which is clear by Lemma 3.3.

Clearly, $S^{0}$ is a bijection by Lemma 3.2.

5.2. Case $n=2$. Now, we construct a (linear) map $S^{2}: C_{b}\left(H^{2}, N_{0}(H, Y)^{* *}\right) \rightarrow$ $C_{b}\left(G^{2}, N_{0}(G, X)^{* *}\right)$.

Write

$$
\begin{aligned}
& X_{i}=\left\{x: c\left(g_{0}^{-1}, x\right)=h_{i}^{-1}\right\}, \\
& X_{j}=\left\{x: c\left(g_{1}^{-1}, x\right)=h_{j}^{-1}\right\}, \\
& X_{k}=\left\{x: c\left(\left(g_{0} g_{1}\right)^{-1}, x\right)=h_{k}^{-1}\right\}, \\
& X_{s}=\left\{x: c\left(\left(g_{1} g_{2}\right)^{-1}, x\right)=h_{s}^{-1}\right\}, \\
& X_{l}=\left\{x: c\left(g_{2}^{-1}, x\right)=h_{l}^{-1}\right\} .
\end{aligned}
$$

Given $f \in C_{b}\left(H^{2}, N_{0}(H, Y)^{* *}\right)$, we define $S^{2}(f)=f^{\prime}$, where for each $\tau \in N_{0}(G, X)^{*}$,

$$
f^{\prime}\left(g_{0}, g_{1}\right)(\tau):=\sum_{i, j}\left\langle f\left(h_{i}, h_{j}\right),\left.\tau\right|_{g_{0} X_{j} \cap X_{i}} \pi\right\rangle
$$

\section{Step 1: $S^{2}$ is well-defined.}

To show $\sup _{\left(g_{0}, g_{1}\right) \in G^{2}}\left\|f^{\prime}\left(g_{0}, g_{1}\right)\right\|<\infty$, apply Lemma 5.1 to $X=\sqcup_{i, j} g_{0} X_{j} \cap X_{i}$.

Step 2: check $\partial^{1} S^{1}=S^{2} \partial^{1}$.

Take any $f \in C_{b}\left(H, N_{0}(H, Y)^{* *}\right)$, write $f^{\prime}=S^{1} f$. For any $g_{1}, g_{2} \in G$ and $\tau \in$ $N_{0}(G, X)^{*}$, we have

$$
\begin{aligned}
& \left(S^{2} \partial^{1} f\right)\left(g_{1}, g_{2}\right)(\tau) \\
= & \sum_{j, l}\left\langle\left(\partial^{1} f\right)\left(h_{j}, h_{l}\right),\left.\tau\right|_{g_{1} X_{l} \cap X_{j}} \pi\right\rangle \quad\left(\text { def. of } S^{2}\right) \\
= & \sum_{j, l}\left\langle h_{j} f\left(h_{l}\right)-f\left(h_{j} h_{l}\right)+f\left(h_{j}\right),\left.\tau\right|_{g_{1} X_{l} \cap X_{j}} \pi\right\rangle \quad\left(\text { def. of } \partial^{1}\right)
\end{aligned}
$$




$$
\begin{aligned}
= & \sum_{j, l}\left\langle h_{j} f\left(h_{l}\right),\left.\tau\right|_{g_{1} X_{l} \cap X_{j}} \pi\right\rangle-\sum_{j, l}\left\langle f\left(h_{j} h_{l}\right),\left.\tau\right|_{g_{1} X_{l} \cap X_{j}} \pi\right\rangle+\sum_{j, l}\left\langle f\left(h_{j}\right),\left.\tau\right|_{g_{1} X_{l} \cap X_{j}} \pi\right\rangle . \\
& \left(\partial^{1} S^{1} f\right)\left(g_{1}, g_{2}\right)(\tau) \\
= & \left(g_{1} f^{\prime}\left(g_{2}\right)-f^{\prime}\left(g_{1} g_{2}\right)+f^{\prime}\left(g_{1}\right)\right)(\tau) \quad\left(\text { def. of } \partial^{1}\right) \\
= & \sum_{l}\left\langle\pi^{* *}\left(f\left(h_{l}\right)\right),\left(g_{1}^{-1} \tau\right)_{l}\right\rangle-\sum_{s}\left\langle\pi^{* *}\left(f\left(h_{s}\right)\right), \tau_{s}\right\rangle+\sum_{j}\left\langle\pi^{* *}\left(f\left(h_{j}\right)\right), \tau_{j}\right\rangle .
\end{aligned}
$$

By comparing the two expressions above, it suffices to show each corresponding terms are equal, i.e.

$$
\begin{aligned}
\sum_{j, l}\left\langle h_{j} f\left(h_{l}\right),\left.\tau\right|_{g_{1} X_{l} \cap X_{j}} \pi\right\rangle & =\sum_{l}\left\langle\pi^{* *}\left(f\left(h_{l}\right)\right),\left(g_{1}^{-1} \tau\right)_{l}\right\rangle, \\
\sum_{j, l}\left\langle f\left(h_{j} h_{l}\right),\left.\tau\right|_{g_{1} X_{l} \cap X_{j}} \pi\right\rangle & =\sum_{s}\left\langle\pi^{* *}\left(f\left(h_{s}\right)\right), \tau_{s}\right\rangle, \\
\sum_{j, l}\left\langle f\left(h_{j}\right),\left.\tau\right|_{g_{1} X_{l} \cap X_{j}} \pi\right\rangle & =\sum_{j}\left\langle\pi^{* *}\left(f\left(h_{j}\right)\right), \tau_{j}\right\rangle .
\end{aligned}
$$

To check (21), it suffices to show for each $l$,

$$
\sum_{j}\left\langle h_{j} f\left(h_{l}\right),\left.\tau\right|_{g_{1} X_{l} \cap X_{j}} \pi\right\rangle=\left\langle\pi^{* *}\left(f\left(h_{l}\right)\right),\left(g_{1}^{-1} \tau\right)_{l}\right\rangle .
$$

Equivalently, we check $\sum_{j} h_{j}^{-1}\left(\left.\tau\right|_{g_{1} X_{l} \cap X_{j}} \pi\right)=\left(g_{1}^{-1} \tau\right){ }_{l} \pi$ holds.

Take any $\xi \in N_{0}(H, Y)$, observe that

$$
\begin{aligned}
\sum_{j} h_{j}^{-1}\left(\left.\tau\right|_{g_{1} X_{l} \cap X_{j}} \pi\right)(\xi) & =\sum_{j}\left(\left.\tau\right|_{g_{1} X_{l} \cap X_{j}} \pi\right)\left(h_{j} \xi\right) \quad \text { (def. of dual action) } \\
& =\sum_{j} \tau\left(\left.\pi\left(h_{j} \xi\right)\right|_{g_{1} X_{l} \cap X_{j}}\right) \\
& \left.=\sum_{j} \tau\left(\left.\left(g_{1} \pi(\xi)\right)\right|_{g_{1} X_{l} \cap X_{j}}\right)\right) \quad \text { (Lemma 3.3) } \\
& \left.=\tau\left(\left.\left(g_{1} \pi(\xi)\right)\right|_{\sqcup_{j} g_{1} X_{l} \cap X_{j}}\right)\right) \\
& =\tau\left(\left.\left(g_{1} \pi(\xi)\right)\right|_{g_{1} X_{l}}\right) \quad\left(X=\sqcup_{j} X_{j}\right) \\
& =\tau\left(g_{1}\left(\left.\pi(\xi)\right|_{X_{l}}\right)\right) \\
& =\left(g_{1}^{-1} \tau\right)_{l} \pi(\xi) .
\end{aligned}
$$

Hence, $\sum_{j} h_{j}^{-1}\left(\left.\tau\right|_{g_{1} X_{l} \cap X_{j}} \pi\right)=\left(g_{1}^{-1} \tau\right)_{l} \pi$ holds.

To check (22), observe that by the cocycle identity, $X_{s}=\sqcup_{(j, l) \in I_{s}}\left(g_{1} X_{l} \cap X_{j}\right)$ holds, where $I_{s}:=\left\{(j, l): h_{s}=h_{j} h_{l}\right\}$.

LHS of (22)

$$
=\sum_{s} \sum_{(j, l) \in I_{s}}\left\langle f\left(h_{j} h_{l}\right),\left.\tau\right|_{g_{1} X_{l} \cap X_{j}} \pi\right\rangle\left(\text { as } g_{1} X_{l} \cap X_{j} \neq \emptyset \text { only if }(j, l) \in I_{s} \text { for some } s\right)
$$




$$
\begin{aligned}
& =\sum_{s} \sum_{(j, l) \in I_{s}}\left\langle f\left(h_{s}\right),\left.\tau\right|_{g_{1} X_{l} \cap X_{j}} \pi\right\rangle \\
& =\sum_{s}\left\langle f\left(h_{s}\right),\left.\tau\right|_{\sqcup_{(j, l) \in I_{s}} g_{1} X_{l} \cap X_{j}} \pi\right\rangle \\
& =\sum_{s}\left\langle f\left(h_{s}\right),\left.\tau\right|_{X_{s}}\right\rangle\left(\text { as } X_{s}=\sqcup_{(j, l) \in I_{s}}\left(g_{1} X_{l} \cap X_{j}\right)\right) \\
& =\sum_{s}\left\langle f\left(h_{s}\right), \tau_{s} \pi\right\rangle=\operatorname{RHS} \text { of }(22) .
\end{aligned}
$$

To check (23), it suffices to show for each $j, \sum_{l}\left\langle f\left(h_{j}\right),\left.\tau\right|_{g_{1} X_{l} \cap X_{j}} \pi\right\rangle=\left\langle\pi^{* *}\left(f\left(h_{j}\right)\right), \tau_{j}\right\rangle$. Observe that

$$
\sum_{l}\left\langle f\left(h_{j}\right),\left.\tau\right|_{g_{1} X_{l} \cap X_{j}} \pi\right\rangle=\left\langle f\left(h_{j}\right),\left.\tau\right|_{\sqcup_{l} g_{1} X_{l} \cap X_{j}} \pi\right\rangle=\left\langle f\left(h_{j}\right),\left.\tau\right|_{X_{j}} \pi\right\rangle=\left\langle\pi^{* *}\left(f\left(h_{j}\right)\right), \tau_{j}\right\rangle .
$$

\section{Step 3: check that $S^{1}$ is a bijection.}

For this purpose, we define a map $T^{1}: C_{b}\left(G, N_{0}(G, X)^{* *}\right) \rightarrow C_{b}\left(H, N_{0}(H, Y)^{* *}\right)$ by setting $T^{1}(z)=z^{\prime}$, where $z^{\prime}(h)(\nu):=\sum_{i}\left\langle L^{* *}\left(z\left(g_{i}\right)\right), \nu_{i}\right\rangle$. Here $\nu \in N_{0}(H, Y)^{*}$, $\nu_{i}=\left.\nu\right|_{Y_{i}}, Y_{i}=\left\{y: c^{\prime}\left(h^{-1}, y\right)=g_{i}^{-1}\right\}$.

Now, we check $T^{1} S^{1}=i d$ holds. $S^{1} T^{1}=i d$ can be checked similarly by symmetry.

Take any $f \in C_{b}\left(H, N_{0}(H, Y)^{* *}\right), h \in H$ and $\nu \in N_{0}(H, Y)^{*}$, write $f^{\prime}=S^{1} f$.

$$
\begin{aligned}
\left(T^{1} S^{1} f\right)(h)(\nu)= & \sum_{i}\left\langle L^{* *}\left(f^{\prime}\left(g_{i}\right)\right), \nu_{i}\right\rangle=\sum_{i}\left\langle f^{\prime}\left(g_{i}\right), \nu_{i} L\right\rangle \\
= & \sum_{i} \sum_{j}\left\langle\pi^{* *}\left(f\left(h_{j}\right)\right),\left(\nu_{i} L\right)_{j}\right\rangle \\
& \left(\text { Here, }\left(\nu_{i} L\right)_{j}:=\left.\left(\nu_{i} L\right)\right|_{X_{g_{i}, h_{j}}}, X_{g_{i}, h_{j}}=\left\{x: c\left(g_{i}^{-1}, x\right)=h_{j}^{-1}\right\} .\right) \\
= & \sum_{i} \sum_{j}\left\langle f\left(h_{j}\right),\left(\nu_{i} L\right)_{j} \pi\right\rangle .
\end{aligned}
$$

Now, we claim that $\left(\nu_{i} L\right)_{j} \pi=\left.\nu\right|_{\phi\left(X_{g_{i}, h_{j}}\right) \cap Y_{i}}$. To see this, take any $\xi \in N_{0}(H, Y)$, then

$$
\begin{aligned}
\left(\nu_{i} L\right)_{j} \pi(\xi) & =\left(\nu_{i} L\right)\left(\left.\pi(\xi)\right|_{X_{g_{i}, h_{j}}}\right)=\nu\left(\left[L \left(\left.\pi(\xi)\right|_{\left.\left.\left.X_{g_{i}, h_{j}}\right)\right]\left.\right|_{Y_{i}}\right)}\right.\right.\right. \\
& =\nu\left(\left.\xi\right|_{\phi\left(X_{g_{i}, h_{j}}\right) \cap Y_{i}}\right) \quad(\text { Lemma 3.4 }) \\
& =\left(\left.\nu\right|_{\phi\left(X_{g_{i}, h_{j}}\right) \cap Y_{i}}\right)(\xi) .
\end{aligned}
$$

Note that $\phi\left(X_{g_{i}, h_{j}}\right) \cap Y_{i}=Y_{i}$ if $h_{j}=h$ and $\emptyset$ otherwise. Therefore,

$$
\left(T^{1} S^{1} f\right)(h)(\nu)=\sum_{i} \sum_{j}\left\langle f\left(h_{j}\right),\left(\nu_{i} L\right)_{j} \pi\right\rangle=\sum_{i}\left\langle f(h), \nu_{i}\right\rangle=\left\langle f(h), \sum_{i} \nu_{i}\right\rangle=f(h)(\nu) .
$$

So, $T^{1} S^{1}=i d$. By symmetry, $S^{1} T^{1}=i d$. 
5.3. Case $n=3$. Now, we construct a (linear) map $S^{3}: C_{b}\left(H^{3}, N_{0}(H, Y)^{* *}\right) \rightarrow$ $C_{b}\left(G^{3}, N_{0}(G, X)^{* *}\right)$. For each $f \in C_{b}\left(H^{3}, N_{0}(H, Y)^{* *}\right)$, define $S^{3}(f)=f^{\prime}$, where

$$
f^{\prime}\left(g_{0}, g_{1}, g_{2}\right)(\tau):=\sum_{i, j, l}\left\langle f\left(h_{i}, h_{j}, h_{l}\right),\left.\tau\right|_{\left(g_{0} g_{1}\right) X_{l} \cap g_{0} X_{j} \cap X_{i}} \pi\right\rangle .
$$

Here, $\tau \in N_{0}(H, Y)^{*}$ and we still use (20) for the definition of $X_{i}, X_{j}, X_{k}, X_{l}, X_{s}$.

\section{Step 1: check $S^{3}$ is well-defined.}

The proof is similar to the one when showing $S^{1}$ is well-defined.

Step 2: check $S^{3} \partial^{2}=\partial^{2} S^{2}$.

Take any $f \in C_{b}\left(H^{3}, N_{0}(H, Y)^{* *}\right)$, write $f^{\prime}=S^{2} f$, we have

$$
\begin{aligned}
& \left(S^{3} \partial^{2} f\right)\left(g_{0}, g_{1}, g_{2}\right)(\tau) \\
= & \sum_{i, j, l}\left\langle\left(\partial^{2} f\right)\left(h_{i}, h_{j}, h_{l}\right),\left.\tau\right|_{\left(g_{0} g_{1}\right) X_{l} \cap g_{0} X_{j} \cap X_{i}} \pi\right\rangle \\
= & \sum_{i, j, l}\left\langle h_{i} f\left(h_{j}, h_{l}\right)-f\left(h_{i} h_{j}, h_{l}\right)+f\left(h_{i}, h_{j} h_{l}\right)-f\left(h_{i}, h_{j}\right),\left.\tau\right|_{\left(g_{0} g_{1}\right) X_{l} \cap g_{0} X_{j} \cap X_{i}} \pi\right\rangle \\
= & \sum_{i, j, l}\left\langle h_{i} f\left(h_{j}, h_{l}\right),\left.\tau\right|_{\left(g_{0} g_{1}\right) X_{l} \cap g_{0} X_{j} \cap X_{i}} \pi\right\rangle-\sum_{i, j, l}\left\langle f\left(h_{i} h_{j}, h_{l}\right),\left.\tau\right|_{\left(g_{0} g_{1}\right) X_{l} \cap g_{0} X_{j} \cap X_{i}} \pi\right\rangle \\
+ & \sum_{i, j, l}\left\langle f\left(h_{i}, h_{j} h_{l}\right),\left.\tau\right|_{\left(g_{0} g_{1}\right) X_{l} \cap g_{0} X_{j} \cap X_{i}} \pi\right\rangle-\sum_{i, j, l}\left\langle f\left(h_{i}, h_{j}\right),\left.\tau\right|_{\left(g_{0} g_{1}\right) X_{l} \cap g_{0} X_{j} \cap X_{i}} \pi\right\rangle \\
= & : \text { (1) - (2) + (3) - (4). }
\end{aligned}
$$

Here, (1), (2), (3) and (4) denote the corresponding terms appeared above.

$$
\begin{aligned}
& \left(\partial^{2} S^{2} f\right)\left(g_{0}, g_{1}, g_{2}\right)(\tau) \\
= & \left(g_{0} f^{\prime}\left(g_{1}, g_{2}\right)-f^{\prime}\left(g_{0} g_{1}, g_{2}\right)+f^{\prime}\left(g_{0}, g_{1} g_{2}\right)-f^{\prime}\left(g_{0}, g_{1}\right)\right)(\tau) \\
= & \sum_{j, l}\left\langle f\left(h_{j}, h_{l}\right),\left.\left(g_{0}^{-1} \tau\right)\right|_{g_{1} X_{l} \cap X_{j}} \pi\right\rangle-\sum_{k, l}\left\langle f\left(h_{k}, h_{l}\right),\left.\tau\right|_{g_{0} g_{1} X_{l} \cap X_{k}} \pi\right\rangle \\
+ & \sum_{i, s}\left\langle f\left(h_{i}, h_{s}\right),\left.\tau\right|_{g_{0} X_{s} \cap X_{i}} \pi\right\rangle-\sum_{i, j}\left\langle f\left(h_{i}, h_{j}\right),\left.\tau\right|_{g_{0} X_{j} \cap X_{i}} \pi\right\rangle \\
= & \text { (5) - (6) + (7) - (8). }
\end{aligned}
$$

Now, in order to prove (1) - (2) + (3) - (4) = (5) - (6) + (7) - (8), it suffices to check the corresponding terms are equal, i.e. (1) $=(5),(2)=(6)$, (3) $=(7)$ and (4) $=(8)$. More precisely, we want to prove the following equalities hold.

$$
\sum_{i, j, l}\left\langle h_{i} f\left(h_{j}, h_{l}\right),\left.\tau\right|_{\left(g_{0} g_{1}\right) X_{l} \cap g_{0} X_{j} \cap X_{i}} \pi\right\rangle=\sum_{j, l}\left\langle f\left(h_{j}, h_{l}\right),\left.\left(g_{0}^{-1} \tau\right)\right|_{g_{1} X_{l} \cap X_{j}} \pi\right\rangle,
$$




$$
\begin{aligned}
\sum_{i, j, l}\left\langle f\left(h_{i} h_{j}, h_{l}\right),\left.\tau\right|_{\left(g_{0} g_{1}\right) X_{l} \cap g_{0} X_{j} \cap X_{i}} \pi\right\rangle & =\sum_{k, l}\left\langle f\left(h_{k}, h_{l}\right),\left.\tau\right|_{g_{0} g_{1} X_{l} \cap X_{k}} \pi\right\rangle, \\
\sum_{i, j, l}\left\langle f\left(h_{i}, h_{j} h_{l}\right),\left.\tau\right|_{\left(g_{0} g_{1}\right) X_{l} \cap g_{0} X_{j} \cap X_{i}} \pi\right\rangle & =\sum_{i, s}\left\langle f\left(h_{i}, h_{s}\right),\left.\tau\right|_{g_{0} X_{s} \cap X_{i}} \pi\right\rangle, \\
\sum_{i, j, l}\left\langle f\left(h_{i}, h_{j}\right),\left.\tau\right|_{\left(g_{0} g_{1}\right) X_{l} \cap g_{0} X_{j} \cap X_{i}} \pi\right\rangle & =\sum_{i, j}\left\langle f\left(h_{i}, h_{j}\right),\left.\tau\right|_{g_{0} X_{j} \cap X_{i}} \pi\right\rangle .
\end{aligned}
$$

To check (24), it suffices to check for each $j, l$,

$$
\sum_{i}\left\langle h_{i} f\left(h_{j}, h_{l}\right),\left.\tau\right|_{\left(g_{0} g_{1}\right) X_{l} \cap g_{0} X_{j} \cap X_{i}} \pi\right\rangle=\left\langle f\left(h_{j}, h_{l}\right),\left.\left(g_{0}^{-1} \tau\right)\right|_{g_{1} X_{l} \cap X_{j}} \pi\right\rangle .
$$

Observe that

$$
h_{i}^{-1}\left(\left.\tau\right|_{\left(g_{0} g_{1}\right) X_{l} \cap g_{0} X_{j} \cap X_{i}} \pi\right)=\left.\left(g_{0}^{-1} \tau\right)\right|_{g_{1} X_{l} \cap X_{j} \cap g_{0}^{-1} X_{i}} \pi .
$$

Indeed, take any $\xi \in N_{0}(H, Y)$, and write $X_{0}:=\left(g_{0} g_{1}\right) X_{l} \cap g_{0} X_{j} \cap X_{i}$, we have $\left(h_{i}^{-1}\left(\left.\tau\right|_{X_{0}} \pi\right)\right) \xi=\tau\left(\left.\pi\left(h_{i} \xi\right)\right|_{X_{0}}\right) \stackrel{\text { Lem.3.3 }}{=} \tau\left(g_{0}\left(\left.\pi(\xi)\right|_{g_{0}^{-1} X_{0}}\right)\right)=\left[\left.\left(g_{0}^{-1} \tau\right)\right|_{g_{0}^{-1} X_{0}} \pi\right](\xi)$.

Therefore,

$$
\begin{aligned}
\text { LHS of }(28) & =\sum_{i}\left\langle h_{i} f\left(h_{j}, h_{l}\right),\left.\tau\right|_{\left(g_{0} g_{1}\right) X_{l} \cap g_{0} X_{j} \cap X_{i}} \pi\right\rangle \\
& =\sum_{i}\left\langle f\left(h_{j}, h_{l}\right), h_{i}^{-1}\left(\left.\tau\right|_{\left(g_{0} g_{1}\right) X_{l} \cap g_{0} X_{j} \cap X_{i}} \pi\right)\right\rangle \\
& \left.\stackrel{(29)}{=} \sum_{i}\left\langle f\left(h_{j}, h_{l}\right),\left.\left(g_{0}^{-1} \tau\right)\right|_{g_{1} X_{l} \cap X_{j} \cap g_{0}^{-1} X_{i}} \pi\right)\right\rangle \\
& =\left\langle f\left(h_{j}, h_{l}\right),\left.\left(g_{0}^{-1} \tau\right)\right|_{\sqcup_{i}\left(g_{1} X_{l} \cap X_{j} \cap g_{0}^{-1} X_{i}\right)} \pi\right\rangle \\
& =\left\langle f\left(h_{j}, h_{l}\right),\left.\left(g_{0}^{-1} \tau\right)\right|_{g_{1} X_{l} \cap X_{j}} \pi\right\rangle \\
& =\operatorname{RHS} \text { of }(28) .
\end{aligned}
$$
So,

To check (25), observe $X_{k}=\sqcup_{(i, j) \in I_{k}} g_{0} X_{j} \cap X_{i}$, where $I_{k}=\left\{(i, j): h_{k}=h_{i} h_{j}\right\}$.

$$
\begin{aligned}
\text { RHS of }(25) & =\sum_{k, l} \sum_{(i, j) \in I_{k}}\left\langle f\left(h_{i} h_{j}, h_{l}\right),\left.\tau\right|_{g_{0} g_{1} X_{l} \cap g_{0} X_{j} \cap X_{i}} \pi\right\rangle \\
& =\sum_{l} \sum_{k} \sum_{(i, j) \in I_{k}}\left\langle f\left(h_{i} h_{j}, h_{l}\right),\left.\tau\right|_{g_{0} g_{1} X_{l} \cap g_{0} X_{j} \cap X_{i}} \pi\right\rangle \\
& =\sum_{l, i, j}\left\langle f\left(h_{i} h_{j}, h_{l}\right),\left.\tau\right|_{g_{0} g_{1} X_{l} \cap g_{0} X_{j} \cap X_{i}} \pi\right\rangle \\
& =\text { LHS of }(25) .
\end{aligned}
$$

Here, the 2nd last equality holds as $g_{0} g_{1} X_{l} \cap g_{0} X_{j} \cap X_{i} \neq \emptyset$ only if $(i, j) \in I_{k}$ for some $k$. 
To check (26), use $X_{s}=\sqcup_{(j, l) \in I_{s}} g_{1} X_{l} \cap X_{j}$, where $I_{s}=\left\{(j, l): h_{s}=h_{j} h_{l}\right\}$ to deduce

$$
\begin{aligned}
\text { RHS of }(26) & =\sum_{i, s}\left\langle f\left(h_{i}, h_{s}\right),\left.\tau\right|_{g_{0} X_{s} \cap X_{i}} \pi\right\rangle \\
& =\sum_{i, s} \sum_{(j, l) \in I_{s}}\left\langle f\left(h_{i}, h_{j} h_{l}\right),\left.\tau\right|_{g_{0} g_{1} X_{l} \cap g_{0} X_{j} \cap X_{i}} \pi\right\rangle \\
& =\sum_{k, l} \sum_{(i, j) \in I_{k}}\left\langle f\left(h_{i}, h_{j} h_{l}\right),\left.\tau\right|_{g_{0} g_{1} X_{l} \cap g_{0} X_{j} \cap X_{i}} \pi\right\rangle \\
& =\sum_{l, i, j}\left\langle f\left(h_{i}, h_{j} h_{l}\right),\left.\tau\right|_{g_{0} g_{1} X_{l} \cap g_{0} X_{j} \cap X_{i}} \pi\right\rangle \\
& =\operatorname{LHS} \text { of }(26) .
\end{aligned}
$$

The 3rd last equality holds since we have a bijection between the index sets:

$$
\left\{(i, s, j, l): h_{s}=h_{j} h_{l}, g_{0} g_{1} X_{l} \cap g_{0} X_{j} \cap X_{i} \neq \emptyset\right\}
$$

and

$$
\left\{(k, l, i, j): h_{k}=h_{i} h_{j}, g_{0} g_{1} X_{l} \cap g_{0} X_{j} \cap X_{i} \neq \emptyset\right\} .
$$

And the 2nd last equality holds as $g_{0} g_{1} X_{l} \cap g_{0} X_{j} \cap X_{i} \neq \emptyset$ only if $(i, j) \in I_{k}$ for some $k$.

To check (27), just observe $g_{0} X_{j} \cap X_{i}=\sqcup_{l}\left(g_{0} g_{1} X_{l} \cap g_{0} X_{j} \cap X_{i}\right)$.

\section{Step 3: $S^{2}$ is a bijection.}

Define $T^{2}: C_{b}\left(G^{2}, N_{0}(G, X)^{* *}\right) \rightarrow C_{b}\left(H^{2}, N_{0}(H, Y)^{* *}\right)$ by setting $T^{2}\left(f^{\prime}\right)=f^{\prime \prime}$, where

$$
f^{\prime \prime}\left(h_{0}, h_{1}\right)(\nu)=\sum_{i, j}\left\langle f^{\prime}\left(g_{i}, g_{j}\right),\left.\nu\right|_{h_{0} Y_{j} \cap Y_{i}} L\right\rangle
$$

Here $\nu \in N_{0}(H, Y)^{*}$, and

$$
\begin{aligned}
& Y_{i}:=\left\{y \in Y ; c^{\prime}\left(h_{0}^{-1}, y\right)=g_{i}^{-1}\right\}, \\
& Y_{j}:=\left\{y \in Y ; c^{\prime}\left(h_{1}^{-1}, y\right)=g_{j}^{-1}\right\} .
\end{aligned}
$$

Now, let us check $T^{2} S^{2}=i d$.

Take any $f \in C_{b}\left(H^{2}, N_{0}(H, Y)^{* *}\right), \nu \in N_{0}(H, Y)^{*}$, let $f^{\prime}=S^{2} f$ and $f^{\prime \prime}=T^{2} f^{\prime}$, we aim to show $f^{\prime \prime}\left(h_{0}, h_{1}\right)(\nu)=f\left(h_{0}, h_{1}\right)(\nu)$.

A calculation shows

$$
\begin{aligned}
f^{\prime \prime}\left(h_{0}, h_{1}\right)(\nu) & =\sum_{i, j} \sum_{s, t}\left\langle f\left(h_{s}, h_{t}\right),\left.\left(\left.\nu\right|_{h_{0} Y_{j} \cap Y_{i}} L\right)\right|_{g_{i} X_{g_{j}, h_{t}} \cap X_{g_{i}, h_{s}}} \pi\right\rangle \\
& =\sum_{i, j} \sum_{s, t}\left\langle f\left(h_{s}, h_{t}\right),\left.\nu\right|_{h_{0} Y_{j} \cap Y_{i} \cap \phi\left(g_{i} X_{g_{j}, h_{t}} \cap X_{g_{i}, h_{s}}\right)}\right\rangle .
\end{aligned}
$$

Here, $X_{g_{i}, h_{s}}=\left\{x \in X: c\left(g_{i}^{-1}, x\right)=h_{s}^{-1}\right\}$ and $X_{g_{j}, h_{t}}=\left\{x \in X: c\left(g_{j}^{-1}, x\right)=h_{t}^{-1}\right\}$. 
One can check that $h_{0} Y_{j} \cap Y_{i} \cap \phi\left(g_{i} X_{g_{j}, h_{t}} \cap X_{g_{i}, h_{s}}\right)=\emptyset$ unless $\left(h_{s}, h_{t}\right)=\left(h_{0}, h_{1}\right)$. If this condition holds, then $h_{0} Y_{j} \cap Y_{i} \cap \phi\left(g_{i} X_{g_{j}, h_{1}} \cap X_{g_{i}, h_{0}}\right)=h_{0} Y_{j} \cap Y_{i}$.

Hence,

$$
f^{\prime \prime}\left(h_{0}, h_{1}\right)(\nu)=\sum_{i, j}\left\langle f\left(h_{0}, h_{1}\right),\left.\nu\right|_{h_{0} Y_{j} \cap Y_{i}}\right\rangle=f\left(h_{0}, h_{1}\right)(\nu)
$$

5.4. General case. We'll define a map $S^{n}: C_{b}\left(H^{n}, N_{0}(H, Y)^{* *}\right) \rightarrow C_{b}\left(G^{n}, N_{0}(G, X)^{* *}\right)$ and check it is a bijective cochain map. Hence, it induces an isomorphism between the two bounded cohomology groups.

We introduce some notations which will be used in this subsection.

Let $g_{0}, \ldots, g_{n}$ be elements in $G$. Write

$$
\begin{aligned}
c\left(g_{j}^{-1}, X\right) & :=\left\{h_{t_{j}}^{-1} \mid t_{j}\right\}, \\
X_{t_{j}} & :=\left\{x \in X: c\left(g_{j}^{-1}, x\right)=h_{t_{j}}^{-1}\right\}, \\
c\left(\left(g_{i} g_{i+1}\right)^{-1}, X\right) & :=\left\{h_{s_{i}}^{-1} \mid s_{i}\right\}, \\
X_{s_{i}} & :=\left\{x \in X: c\left(\left(g_{i} g_{i+1}\right)^{-1}, x\right)=h_{s_{i}}^{-1}\right\} .
\end{aligned}
$$

Note that $X_{s_{i}}=\sqcup_{\left(t_{i}, t_{i+1}\right) \in \Delta_{i}} X_{t_{i}} \cap g_{i} X_{t_{i+1}}$, where $\Delta_{i}:=\left\{\left(t_{i}, t_{i+1}\right): h_{s_{i}}=h_{t_{i}} h_{t_{i+1}}\right\}$.

We remind the reader once again that $X_{t_{j}}, X_{s_{i}}$ and $\Delta_{i}$ are just simplified versions for the notations $X_{g_{j}, h_{t_{j}}}, X_{g_{i} g_{i+1}, h_{s_{i}}}$ and $\Delta_{s_{i}}$ respectively, which we will switch to if necessary.

Let $f \in C_{b}\left(H^{n}, N_{0}(H, Y)^{* *}\right)$, we define $S^{n}(f):=f^{\prime} \in C_{b}\left(G^{n}, N_{0}(G, X)^{* *}\right)$, where

$$
f^{\prime}\left(g_{0}, \ldots, g_{n-1}\right)(\tau):=\sum_{t_{0}, \ldots, t_{n-1}}\left\langle f\left(h_{t_{0}}, \ldots, h_{t_{n-1}}\right),\left.\tau\right|_{\left[t_{0}, \ldots, t_{n-1}\right]} \circ \pi\right\rangle
$$

for all $g_{i} \in G$ and $\tau \in N_{0}(G, X)^{*}$.

Here,

$$
\left[t_{0}, \ldots, t_{n-1}\right]:=X_{t_{0}} \cap g_{0} X_{t_{1}} \cap g_{0} g_{1} X_{t_{2}} \cap \cdots \cap\left(g_{0} \cdots g_{n-2}\right) X_{t_{n-1}} .
$$

We split the proof into several steps.

\section{Step 1: $S^{n}$ is well-defined.}

Apply Lemma 5.1 to $X=\sqcup_{t_{0}, \ldots, t_{n-1}}\left[t_{0}, \ldots, t_{n-1}\right]$.

Step 2: $S^{n}$ is a cochain map, i.e. $S^{n+1} \partial^{n}=\partial^{n} S^{n}$.

Fix $f \in C_{b}\left(H^{n}, N_{0}(H, Y)^{* *}\right)$, let $f^{\prime}=S^{n} f$. We aim to show $S^{n+1} \partial^{n} f=\partial^{n} f^{\prime}$. Take any $g_{0}, \ldots, g_{n}$ in $G$, and any $\tau \in N_{0}(G, X)^{*}$. We compute as follows. 
First,

$$
\begin{aligned}
& \partial^{n} f^{\prime}\left(g_{0}, \ldots, g_{n}\right)(\tau) \\
= & {\left[g_{0} f^{\prime}\left(g_{1}, \ldots, g_{n}\right)+\sum_{i=1}^{n}(-1)^{i} f^{\prime}\left(g_{0}, \ldots, g_{i-1} g_{i}, \ldots, g_{n}\right)+(-1)^{n+1} f^{\prime}\left(g_{0}, \ldots, g_{n-1}\right)\right](\tau) } \\
= & \sum_{t_{1}, \ldots, t_{n}}\left\langle f\left(h_{t_{1}}, \ldots, h_{t_{n}}\right),\left.\left(g_{0}^{-1} \tau\right)\right|_{\left[t_{1}, \ldots, t_{n}\right]} \pi\right\rangle \\
& +\sum_{i=1}^{n}(-1)^{i} \sum_{\substack{t_{0}, \ldots, t_{i-2}, s_{i-1}, t_{i+1}, \ldots, t_{n}}}\left\langle f\left(h_{t_{0}}, \ldots, h_{t_{i-2}}, h_{s_{i-1}}, h_{t_{i+1}}, \ldots, h_{t_{n}}\right),\left.\tau\right|_{\left[t_{0}, \ldots, t_{i-2}, s_{i-1}, t_{i+1}, \ldots, t_{n}\right]} \pi\right\rangle \\
& +(-1)^{n+1} \sum_{t_{0}, \ldots, t_{n-1}}\left\langle f\left(h_{t_{0}}, \ldots, h_{t_{n-1}}\right),\left.\tau\right|_{\left[t_{0}, \ldots, t_{n-1}\right]} \pi\right\rangle .
\end{aligned}
$$

Recall here, $c\left(g_{i-1} g_{i}, X\right)=\left\{h_{s_{i-1}} \mid s_{i-1}\right\}, X_{s_{i-1}}=\left\{x: c\left(\left(g_{i-1} g_{i}\right)^{-1}, x\right)=h_{s_{i-1}}^{-1}\right\}$ and

$$
\begin{aligned}
{\left[t_{0}, \ldots, t_{i-2}, s_{i-1}, t_{i+1}, \ldots, t_{n}\right] } & =X_{t_{0}} \cap g_{0} X_{t_{1}} \cap \cdots \cap\left(g_{0} \cdots g_{i-2}\right) X_{s_{i-1}} \\
& \cap\left(g_{0} \cdots g_{i-2} g_{i-1} g_{i}\right) X_{t_{i+1}} \cap \cdots \cap\left(g_{0} \ldots g_{n-1}\right) X_{t_{n}}
\end{aligned}
$$

Second,

$$
\begin{aligned}
& \left(S^{n+1} \partial^{n} f\right)\left(g_{0}, \ldots, g_{n}\right)(\tau) \\
= & \sum_{t_{0}, \ldots, t_{n}}\left\langle\partial^{n}(f)\left(h_{t_{0}}, \ldots, h_{t_{n}}\right),\left.\tau\right|_{\left[t_{0}, \ldots, t_{n}\right]} \pi\right\rangle \\
= & \sum_{t_{0}, \ldots, t_{n}}\left\langle h_{t_{0}} f\left(h_{t_{1}}, \ldots, h_{t_{n}}\right),\left.\tau\right|_{\left[t_{0}, \ldots, t_{n}\right]} \pi\right\rangle \\
& +\sum_{t_{0}, \ldots, t_{n}}\left\langle\sum_{i=1}^{n}(-1)^{i} f\left(h_{t_{0}}, \ldots, h_{t_{i-1}} h_{t_{i}}, \ldots, h_{t_{n}}\right),\left.\tau\right|_{\left[t_{0}, \ldots, t_{n}\right]} \pi\right\rangle \\
& +\sum_{t_{0}, \ldots, t_{n}}\left\langle(-1)^{n+1} f\left(h_{t_{0}}, \ldots, h_{t_{n-1}}\right),\left.\tau\right|_{\left[t_{0}, \ldots, t_{n}\right]} \pi\right\rangle .
\end{aligned}
$$

By comparing (34) with (32), it suffices to show that the corresponding terms are equal, i.e. we want to establish the following identities.

(a)

$$
\sum_{t_{1}, \ldots, t_{n}}\left\langle f\left(h_{t_{1}}, \ldots, h_{t_{n}}\right),\left.\left(g_{0}^{-1} \tau\right)\right|_{\left[t_{1}, \ldots, t_{n}\right]} \pi\right\rangle=\sum_{t_{0}, \ldots, t_{n}}\left\langle h_{t_{0}} f\left(h_{t_{1}}, \ldots, h_{t_{n}}\right),\left.\tau\right|_{\left[t_{0}, \ldots, t_{n}\right]} \pi\right\rangle .
$$

(b) For each $1 \leq i \leq n$,

$$
\begin{aligned}
\sum_{\substack{t_{0}, \ldots, t_{i-2}, s_{i-1}, t_{i+1}, \ldots, t_{n}}}\left\langle f\left(h_{t_{0}}, \ldots, h_{t_{i-2}}, h_{s_{i-1}}, h_{t_{i+1}}, \ldots, h_{t_{n}}\right),\left.\tau\right|_{\left[t_{0}, \ldots, t_{i-2}, s_{i-1}, t_{i+1}, \ldots, t_{n}\right]} \pi\right\rangle \\
=\sum_{t_{0}, \ldots, t_{n}}\left\langle f\left(h_{t_{0}}, \ldots, h_{t_{i-1}} h_{t_{i}}, \ldots, h_{t_{n}}\right),\left.\tau\right|_{\left[t_{0}, \ldots, t_{n}\right]} \pi\right\rangle .
\end{aligned}
$$


(c)

$\sum_{t_{0}, \ldots, t_{n-1}}\left\langle f\left(h_{t_{0}}, \ldots, h_{t_{n-1}}\right),\left.\tau\right|_{\left[t_{0}, \ldots, t_{n-1}\right]} \pi\right\rangle=\sum_{t_{0}, \ldots, t_{n}}\left\langle(-1)^{n+1} f\left(h_{t_{0}}, \ldots, h_{t_{n-1}}\right),\left.\tau\right|_{\left[t_{0}, \ldots, t_{n}\right]} \pi\right\rangle$.

Proof of (a). By Lemma 3.4, $\left.\left(g_{0}^{-1} \tau\right)\right|_{g_{0}^{-1} X_{t_{0}} \cap\left[t_{1}, \ldots, t_{n}\right]} \pi=h_{t_{0}}^{-1}\left(\left.\tau\right|_{\left[t_{0}, \ldots, t_{n}\right]} \pi\right)$ holds.

Indeed, evaluate both sides at any $\xi$ in $N_{0}(H, Y)$, this boils down to check $X_{t_{0}} \cap$ $g_{0}\left[t_{1}, \ldots, t_{n}\right]=\left[t_{0}, \ldots, t_{n}\right]$, which is clear from the definition of $\left[t_{0}, \ldots, t_{n}\right]$.

Using this identity, we deduce

$$
\begin{aligned}
& \text { RHS of (a) } \\
= & \sum_{t_{0}, \ldots, t_{n}}\left\langle f\left(h_{t_{1}}, \ldots, h_{t_{n}}\right), h_{t_{0}}^{-1}\left(\left.\tau\right|_{\left[t_{0}, \ldots, t_{n}\right]} \pi\right)\right\rangle \\
= & \sum_{t_{0}, \ldots, t_{n}}\left\langle f\left(h_{t_{1}}, \ldots, h_{t_{n}}\right),\left.\left(g_{0}^{-1} \tau\right)\right|_{g_{0}^{-1} X_{t_{0}} \cap\left[t_{1}, \ldots, t_{n}\right]} \pi\right\rangle \\
= & \sum_{t_{1}, \ldots, t_{n}}\left\langle f\left(h_{t_{1}}, \ldots, h_{t_{n}}\right),\left.\left(g_{0}^{-1} \tau\right)\right|_{\sqcup_{t_{0}} g_{0}^{-1} X_{t_{0}} \cap\left[t_{1}, \ldots, t_{n}\right]} \pi\right\rangle \\
= & \sum_{t_{1}, \ldots, t_{n}}\left\langle f\left(h_{t_{1}}, \ldots, h_{t_{n}}\right),\left.\left(g_{0}^{-1} \tau\right)\right|_{\left[t_{1}, \ldots, t_{n}\right]} \pi\right\rangle \\
= & \operatorname{LHS} \text { of }(\mathrm{a}) .
\end{aligned}
$$

Proof of (b). From $X_{s_{i-1}}=\sqcup_{\left(t_{i-1}, t_{i}\right) \in \Delta_{i-1}} X_{t_{i-1}} \cap g_{i-1} X_{t_{i}}$, we deduce that

$$
\left[t_{0}, \ldots, t_{i-2}, s_{i-1}, t_{i+1}, \ldots, t_{n}\right]=\sqcup_{\left(t_{i-1}, t_{i}\right) \in \Delta_{i-1}}\left[t_{0}, \ldots, t_{n}\right] .
$$

Indeed, just compare definition (31) and (33).

Hence,

$$
\begin{aligned}
& \sum_{s_{i-1}}\left\langle f\left(h_{t_{0}}, \ldots, h_{t_{i-2}}, h_{s_{i-1}}, h_{t_{i+1}}, \ldots, h_{t_{n}}\right),\left.\tau\right|_{\left[t_{0}, \ldots, t_{i-2}, s_{i-1}, t_{i+1}, \ldots, t_{n}\right]} \pi\right\rangle \\
\stackrel{(35)}{=} & \sum_{s_{i-1}} \sum_{\left(t_{i-1}, t_{i}\right) \in \Delta_{i-1}}\left\langle f\left(h_{t_{0}}, \ldots, h_{t_{i-2}}, h_{t_{i-1}} h_{t_{i}}, h_{t_{i+1}}, \ldots, h_{t_{n}}\right),\left.\tau\right|_{\left[t_{0}, \ldots, t_{n}\right]} \pi\right\rangle \\
= & \sum_{t_{i-1}, t_{i}}\left\langle f\left(h_{t_{0}}, \ldots, h_{t_{i-2}}, h_{t_{i-1}} h_{t_{i}}, h_{t_{i+1}}, \ldots, h_{t_{n}}\right),\left.\tau\right|_{\left[t_{0}, \ldots, t_{n}\right]} \pi\right\rangle .
\end{aligned}
$$

The last equality holds since $\left[t_{0}, \ldots, t_{n}\right] \neq \emptyset$ only if $\left(t_{i-1}, t_{i}\right) \in \Delta_{i-1}$ for some $s_{i-1}$. (Recall $\Delta_{i-1}=\Delta_{s_{i-1}}$ for simplicity.)

Therefore, (b) holds by taking sum over $t_{0}, \ldots, t_{i-2}, t_{i+1}, \ldots, t_{n}$ on both sides of the above equality.

Proof of $(c)$. As $\left[t_{0}, \ldots, t_{n}\right]=\left[t_{0}, \ldots, t_{n-1}\right] \cap\left(g_{0} \cdots g_{n-1}\right) X_{t_{n}}$, we get

$$
\text { RHS of (c) }
$$

$$
\begin{aligned}
& =\sum_{t_{0}, \ldots, t_{n}}\left\langle f\left(h_{t_{0}}, \ldots, h_{t_{n-1}}\right),\left.\tau\right|_{\left[t_{0}, \ldots, t_{n-1}\right] \cap\left(g_{0} \cdots g_{n-1}\right) X_{t_{n}}} \pi\right\rangle \\
& =\sum_{t_{0}, \ldots, t_{n-1}}\left\langle f\left(h_{t_{0}}, \ldots, h_{t_{n-1}}\right),\left.\tau\right|_{\left[t_{0}, \ldots, t_{n-1}\right] \cap \sqcup_{t_{n}}\left(g_{0} \cdots g_{n-1}\right) X_{t_{n}}} \pi\right\rangle
\end{aligned}
$$




$$
\begin{aligned}
& =\sum_{t_{0}, \ldots, t_{n-1}}\left\langle f\left(h_{t_{0}}, \ldots, h_{t_{n-1}}\right),\left.\tau\right|_{\left[t_{0}, \ldots, t_{n-1}\right]} \pi\right\rangle \\
& =\text { LHS of (c). }
\end{aligned}
$$

\section{Step 3: $S^{n}$ is a bijection.}

To check $S^{n}$ is a bijection, consider the natural inverse map $T^{n}$ defined by symmetry as follows.

For $f^{\prime} \in C_{b}\left(G^{n}, N_{0}(G, X)^{* *}\right)$, define $f^{\prime \prime}:=T\left(f^{\prime}\right)$ as follows:

$$
f^{\prime \prime}\left(h_{0}, \ldots, h_{n-1}\right)(\nu):=\sum_{i_{0}, \ldots, i_{n-1}}\left\langle f^{\prime}\left(g_{i_{0}}, \ldots, g_{i_{n-1}}\right),\left.\nu\right|_{\left[i_{0}, \ldots, i_{n-1}\right]} \circ L\right\rangle .
$$

Here, $\nu \in N_{0}(H, Y)^{*},\left[i_{0}, \ldots, i_{n-1}\right]:=Y_{i_{0}} \cap h_{0} Y_{i_{1}} \cap \cdots \cap\left(h_{0} \cdots h_{n-2}\right) Y_{i_{n-1}}$ and $Y_{i_{j}}:=\left\{y: c^{\prime}\left(h_{j}^{-1}, y\right)=g_{i_{j}}^{-1}\right\}$.

Let $f \in C_{b}\left(H^{n}, N_{0}(H, Y)^{* *}\right)$, write $f^{\prime}=S^{n} f$ and $f^{\prime \prime}=T^{n} f^{\prime}$. We aim to show $T^{n} S^{n}=i d$, i.e. $f^{\prime \prime}=f$. Take any $h_{0}, \ldots, h_{n-1}$ in $H$ and any $\nu$ in $N_{0}(H, Y)^{*}$, then

$$
\begin{aligned}
& f^{\prime \prime}\left(h_{0}, \ldots, h_{n-1}\right)(\nu) \\
= & \sum_{i_{0}, \ldots, i_{n-1}}\left\langle f^{\prime}\left(g_{i_{0}}, \ldots, g_{i_{n-1}}\right),\left.\nu\right|_{\left[i_{0}, \ldots, i_{n-1}\right]} \circ L\right\rangle \\
= & \sum_{i_{0}, \ldots, i_{n-1}} \sum_{t_{0}, \ldots, t_{n-1}}\left\langle f\left(h_{t_{0}}, \ldots, h_{t_{n-1}}\right),\left.\left(\left.\nu\right|_{\left[i_{0}, \ldots, i_{n-1}\right]} \circ L\right)\right|_{\left[t_{0}, \ldots, t_{n-1}\right]} \pi\right\rangle .
\end{aligned}
$$

Here, $\left[t_{0}, \ldots, t_{n-1}\right]:=X_{g_{i_{0}}, h_{t_{0}}} \cap g_{i_{0}} X_{g_{i_{1}}, h_{t_{1}}} \cap \cdots \cap\left(g_{i_{0}} \cdots g_{i_{n-2}}\right) X_{g_{i_{n-1}}, h_{t_{n-1}}}$ and $X_{g_{i_{j}}, h_{t_{j}}}:=$ $\left\{x: c\left(g_{i_{j}}^{-1}, x\right)=h_{t_{j}}^{-1}\right\}$.

To continue the proof, we notice the following facts.

$\left(\right.$ Fact 5) $\left.\left(\left.\nu\right|_{\left[i_{0}, \ldots, i_{n-1}\right]} \circ L\right)\right|_{\left[t_{0}, \ldots, t_{n-1}\right]} \pi=\left.\nu\right|_{\left[i_{0}, \ldots, i_{n-1}\right] \cap \phi\left(\left[t_{0}, \ldots, t_{n-1}\right]\right)}$.

To prove this fact, take any $\xi \in N_{0}(H, Y), h \in H$ and $y \in Y$. Let $\xi^{\prime}=$ $\pi(\xi)$ and $\eta=\left.\xi^{\prime}\right|_{\left[t_{0}, \ldots, t_{n-1}\right]}$. We aim to prove $\left(\left.\left(\left.\nu\right|_{\left[i_{0}, \ldots, i_{n-1}\right]} \circ L\right)\right|_{\left[t_{0}, \ldots, t_{n-1}\right]} \pi\right)(\xi)=$ $\left(\left.\nu\right|_{\left[i_{0}, \ldots, i_{n-1}\right] \cap \phi\left(\left[t_{0}, \ldots, t_{n-1}\right]\right)}\right)(\xi)$; equivalently, $\nu\left(\left.(L \circ \eta)\right|_{\left[i_{0}, \ldots, i_{n-1}\right]}\right)=\nu\left(\left.\xi\right|_{\left[i_{0}, \ldots, i_{n-1}\right] \cap \phi\left(\left[t_{0}, \ldots, t_{n-1}\right]\right)}\right)$. This is clear by Lemma 3.4.

(Fact 6$)\left[i_{0}, \ldots, i_{n-1}\right] \cap \phi\left(\left[t_{0}, \ldots, t_{n-1}\right]\right)=\emptyset$ unless $h_{t_{j}}=h_{j}$ for all $0 \leq j \leq n-1$. When these conditions hold, the intersection equals $\left[i_{0}, \ldots, i_{n-1}\right]$.

This fact can be directly checked using cocycle identity and Lemma 3.1.

Now we can continue the computation in (36) as follows.

$$
\begin{aligned}
& f^{\prime \prime}\left(h_{0}, \ldots, h_{n-1}\right)(\nu) \\
& \stackrel{\text { Fact }}{=} \sum_{i_{0}, \ldots, i_{n-1}} \sum_{t_{0}, \ldots, t_{n-1}}\left\langle f\left(h_{t_{0}}, \ldots, h_{t_{n-1}}\right),\left.\nu\right|_{\left[i_{0}, \ldots, i_{n-1}\right] \cap \phi\left(\left[t_{0}, \ldots, t_{n-1}\right]\right)}\right\rangle \\
& \stackrel{\text { Fact }}{=} \sum_{i_{0}, \ldots, i_{n-1}}\left\langle f\left(h_{0}, \ldots, h_{n-1}\right),\left.\nu\right|_{\left[i_{0}, \ldots, i_{n-1}\right]}\right\rangle \\
&=\left\langle f\left(h_{0}, \ldots, h_{n-1}\right), \nu\right\rangle .
\end{aligned}
$$


Therefore, $f^{\prime \prime}=f$ and the proof is finished.

\section{Concluding Remarks}

We end this paper with several remarks addressing various issues related to our main theorems.

(1) It is routine to check that the isomorphism $S$ constructed in the proof preserves the Johnson classes [3, Definition 20] and the fundamental classes [4, Definition 3] for the two actions, which were introduced to study amenability of group actions. Since the triviality of the Johnson class [3, Theorem 1] and the nontriviality of the fundamental class [4, Theorem 9] are used to characterize the amenability of an action, we see that for two topologically free actions, topologically amenability is an invariant property under continuous orbit equivalence.

(2) Under the same assumptions as in our theorems, it is natural to ask whether $H_{n}^{u f}(G \curvearrowright X) \cong H_{n}^{u f}(H \curvearrowright Y)$ for $n \geq 1$ holds. For this question, our method does not work. Indeed, we use crucially the decomposition of elements in $N_{0}(G, X)$ as a finite sum of elements in $N_{0}(G, X)$ with respect to a finite partition of $X$ into clopen subsets. The summands do not belong to $W_{0}(G, X)$ anymore if we decompose an element in $W_{0}(G, X)$. This may be interpreted as saying $W_{0}(G, X)$ is not resinvariant (w.r.t. $X$ ), following [15, Definition 4.1] in spirit or reflecting the fact $W_{0}(G, X)$ is not a $(G, X)$-module in the sense of [17].

In fact, the following suggests the above isomorphism may fail in general. First, by [4, Corollary 5], we know that for a topologically amenable action $G \curvearrowright X, H_{n}^{u f}(G \curvearrowright$ $X) \cong H_{n}(G, \mathbb{R}) \oplus H_{n}\left(G, N_{0}(G, X)^{*}\right)$ holds. Then, we consider two continuous actions which are both topologically amenable and topologically free, e.g. left translation actions on the Stone-Cech compactifications of free groups with different rank, say $F_{2}$ and $F_{3}$. As $F_{2}$ is quasi-isometric to $F_{3}$, we know they are also bilipschitz equivalent by [21]; equivalently (see [15, Corollary 2.21]), $F_{2} \curvearrowright \beta F_{2} \stackrel{\text { coe }}{\sim} F_{3} \curvearrowright \beta F_{3}$. On the one hand, $H_{1}\left(F_{k}, \mathbb{R}\right) \cong \mathbb{R}^{k}$ for all $k \geq 1$ implies $H_{1}\left(F_{2}, \mathbb{R}\right) \approx H_{1}\left(F_{3}, \mathbb{R}\right)$, but on the other hand, $H_{1}\left(F_{2}, N_{0}\left(F_{2}, \beta F_{2}\right)^{*}\right) \cong H_{1}\left(F_{3}, N_{0}\left(F_{3}, \beta F_{3}\right)^{*}\right)$ by our main theorems. This suggests that for the above example, one expects these two actions have nonisomorphic $H_{1}^{u f}$.

Nevertheless, under certain assumptions, we can still have some positive result.

Corollary 6.1. Let $G \curvearrowright X$ and $H \curvearrowright Y$ be topologically free actions which are COE. If $G \curvearrowright X$ is topologically amenable and both $G$ and $H$ are finitely generated torsion free nilpotent groups with finite cohomological dimension, then $H_{i}^{u f}(G \curvearrowright$ $X) \cong H_{i}^{u f}(H \curvearrowright Y)$ for all $i \geq 0$.

Proof. First, observe that $H \curvearrowright Y$ is also topologically amenable. This is clear by previous Remark (1). One can also check this using [4, Definition 7] and the map $\pi$ defined in Lemma 3.2.

Then by [4, Corollary 5], for topologically amenable actions, we have $H_{i}^{u f}(G \curvearrowright$ $X) \cong H_{i}(G, \mathbb{R}) \oplus H_{i}\left(G, N_{0}(G, X)^{*}\right)$. The same holds for the action $H \curvearrowright Y$. 
By Theorem 1.1, it suffices to show that $H_{i}(G, \mathbb{R}) \cong H_{i}(H, \mathbb{R})$.

Observe that COE between the above two actions implies $G$ and $H$ are quasiisometric. Indeed, just fix any $x \in X$, it is easy to check $g \in G \rightarrow H \ni c(g, x)$ is a quasi-isometry using Lemma 3.1.

Now, as $G$ and $H$ are quasi-isometric nilpotent groups, we deduce $H^{i}(G, \mathbb{R}) \cong$ $H^{i}(H, \mathbb{R})$ by $[20$, Theorem 1.5]. Moreover, $c d(G)=c d(H)$ by $[20$, Theorem 1.2] or [15, Corollary 4.42]. The proof is finished by noticing that for finitely generated torsion free nilpotent groups, they are orientable Poincaré duality groups, which implies that $H^{i}(G, \mathbb{R}) \cong H_{n-i}(G, \mathbb{R})$, where $n=c d(G)$. And the same holds for $H$. (For the above assertion and the definition of orientable Poincaré duality groups, see Section 10, Chapter VIII in [6], in particular, [6, Example 1, p. 222].)

(3) (Co)homology groups associated to many coefficient modules are proved to be invariants under COE for two topologically free actions in [15, Theorem 3.1, 3.5], but as far as we can see, the (co)homology groups considered in our paper are not covered by these theorems. It seems plausible one may also prove our theorems using the method in [15], i.e. try to interpret the (co)homologies for $G$ as (co)homologies for the transformation groupoid, but one may need to extend unitary representations of étale locally compact groupoid $([15, \S 3.2],[19])$ to linear isometric representations on Banach spaces. In fact, for two COE topologically free actions $G \curvearrowright X$ and $H \curvearrowright Y$, it may be possible to show there is a one to one correspondence between $(G, X)$-modules of type $M$ in the sense of [17], say $E$, and $(H, Y)$-modules of type $M$, say $F$, and under this correspondence, $H_{b}^{*}\left(G, E^{*}\right) \cong H_{b}^{*}\left(H, F^{*}\right)$. Theorem 1.2 may be thought of as an evidence for this.

Acknowledgement: We thank Prof. Piotr Nowak for helpful discussion related to this paper. We are also very grateful to the anonymous referee. He/She provided us with many valuable suggestions which greatly improved the readability of the paper.

\section{REFERENCES}

[1] C. Anantharaman-Delaroche and J. Renault, Amenable groupoids, Monographies de L'Enseignement Mathématique [Monographs of L'Enseignement Mathématique], vol. 36, L'Enseignement Mathématique, Geneva, 2000. With a foreword by Georges Skandalis and Appendix B by E. Germain.

[2] J. Block and S. Weinberger, Aperiodic tilings, positive scalar curvature and amenability of spaces, J. Amer. Math. Soc. 5 (1992), no. 4, 907-918.

[3] J. Brodzki, G. Niblo, P. Nowak, and N. Wright, Amenable actions, invariant means and bounded cohomology, Journal of Topology and Analysis 4 (2012), no. 3, 321-334.

[4] - A homological characterization of topological amenability, Algebr. Geom. Topol. 12 (2012), no. 3, 1767-1780.

[5] J. Brodzki, G. Niblo, and N. Wright, Pairings, duality, amenability and bounded cohomology, J. Eur. Math. Soc. 14 (2012), no. 5, 1513-1518.

[6] K. Brown, Cohomology of groups, Graduate Texts in Mathematics, vol. 87, Springer-Verlag, New York-Berlin, 1982. 
[7] D. Cohen, Continuous cocycle superrigidity for the full shift over a finitely generated torsion group, IMRN, in press.

[8] N.-P. Chung and Y. Jiang, Continuous cocycle superrigidity for shifts and groups with one end, Math. Ann. 368 (2017), no. 3-4, 1109-1132.

[9] _ Divergence, Undistortion and Hölder Continuous Cocycle Superrigidity for Full Shifts, arXiv: 1709.10218.

[10] E. Guentner and J. Kaminker, Exactness and the Novikov conjecture, Topology 41 (2002), no. 2, 411-418.

[11] N. Higson and J. Roe, Amenable group actions and the Novikov conjecture, J. Reine Angew. Math. 519 (2000), 143-153.

[12] Y. Jiang, Continuous cocycle superrigidity for coinduced actions and relative ends, Proc. Amer. Math. Soc. 147 (2019), no. 1, 315-326.

[13] B. Johnson, Cohomology in Banach algebras, American Mathematical Society, Providence, R.I., 1972. Memoirs of the American Mathematical Society, No. 127.

[14] X. Li, Continuous orbit equivalence rigidity, Ergodic Theory Dynam. Systems 38 (2018), no. 4, $1543-1563$.

[15] _ Dynamical characterizations of quasi-isometry, and applications to cohomology, Algebr. Geom. Topol. 18 (2018), no. 6, 3477-3535.

[16] N. Monod, Continuous bounded cohomology of locally compact groups, Lecture Notes in Mathematics, vol. 1758, Springer-Verlag, Berlin, 2001.

[17] _ A note on topological amenability, IMRN 17 (2011), 3872-3884.

[18] N. Ozawa, Amenable actions and exactness for discrete groups, C. R. Acad. Sci. Paris Sér. I Math. 330 (2000), no. 8, 691-695.

[19] J. Renault, A groupoid approach to $C^{*}$-algebras, Lecture Notes in Mathematics, vol. 793, Springer, Berlin, 1980.

[20] R. Sauer, Homological invariants and quasi-isometry, Geom. Funct. Anal. 16 (2006), no. 2, 476-515.

[21] K. Whyte, Amenability, bi-Lipschitz equivalence, and the von Neumann conjecture, Duke Math. J. 99 (1999), no. 1, 93-112.

Institute of Mathematics, Polish Academy of Sciences, Warsaw, 00-656, Poland. E-mail address: yjiang@impan.pl 